Supporting Information

\title{
Clustering of Death Receptor for Apoptosis Using Nanoscale Patterns of Peptides
}

\author{
Yang Wang, Igor Baars, Ferenc Fördös, Björn Högberg*
}

Department of Medical Biochemistry and Biophysics, Karolinska Institutet, SE-17177

Stockholm, Sweden

*Correspondence to: bjorn.hogberg@ki.se

\begin{tabular}{|l|l|}
\hline Index & Contents \\
\hline Figure S1 & Design of L-structure \\
\hline Figure S2 & Computational 3D structure of L designs \\
\hline Figure S3 & Agarose gel electrophoresis of W and L \\
\hline Figure S4 & Basic structural information of the peptide and TRAIL \\
\hline Figure S5 & Scheme of peptide-DNA conjugation \\
\hline Figure S6 & Conjugates gel electrophoresis followed by silver staining \\
\hline Figure S7 & Current interpretation of apoptosis-driving hexagonal DR5 clusters \\
\hline Figure S8 & Illustration on pattern designs on W \\
\hline Figure S9 & Design of the template for L6 \\
\hline Figure S10 & Design of the template for L11 \\
\hline Figure S11 & Nucleotide-to-nucleotide distance measurement through oxDNA \\
\hline Figure S12 & Agarose gel electrophoresis of peptide-attached W structures \\
\hline Figure S13 & Agarose gel electrophoresis of peptide-attached L structures \\
\hline Figure S14 & Pre-processing of DNA-PAINT data \\
\hline Figure S15 & Plots of localization properties and their respective cutoffs for filtering \\
\hline Figure S16 & Detection of DNA origami ROI coordinates from DNA-PAINT data \\
\hline Figure S17 & Detection of peptides in origami ROIs extracted from DNA-PAINT data \\
\hline Figure S18 & DNA-PAINT images of W37 structures \\
\hline Figure S19 & DNA-PAINT images of W26 structures \\
\hline Figure S20 & DNA-PAINT images of W19 structures \\
\hline Figure S21 & DNA-PAINT images of W16 structures \\
\hline Figure S22 & DNA-PAINT images of W9 structures \\
\hline Figure S23 & DNA-PAINT images of W6 structures \\
\hline Figure S24 & Protruding DNA site occupancy calculated from DNA-PAINT \\
\hline Figure S25 & Inter-peptide distance distribution from DNA-PAINT \\
\hline Figure S26 & Gel-based peptide quantification of L6 \\
\hline Figure S27 & DR5 clusters induced by L6 attached with different number of peptides \\
\hline Figure S28 & Cleaved caspase-8 induced by L6 attached with different number of peptides \\
\hline Table S1 & The half maximal inhibitory concentration (IC 50 of peptide \\
\hline & \\
\hline
\end{tabular}




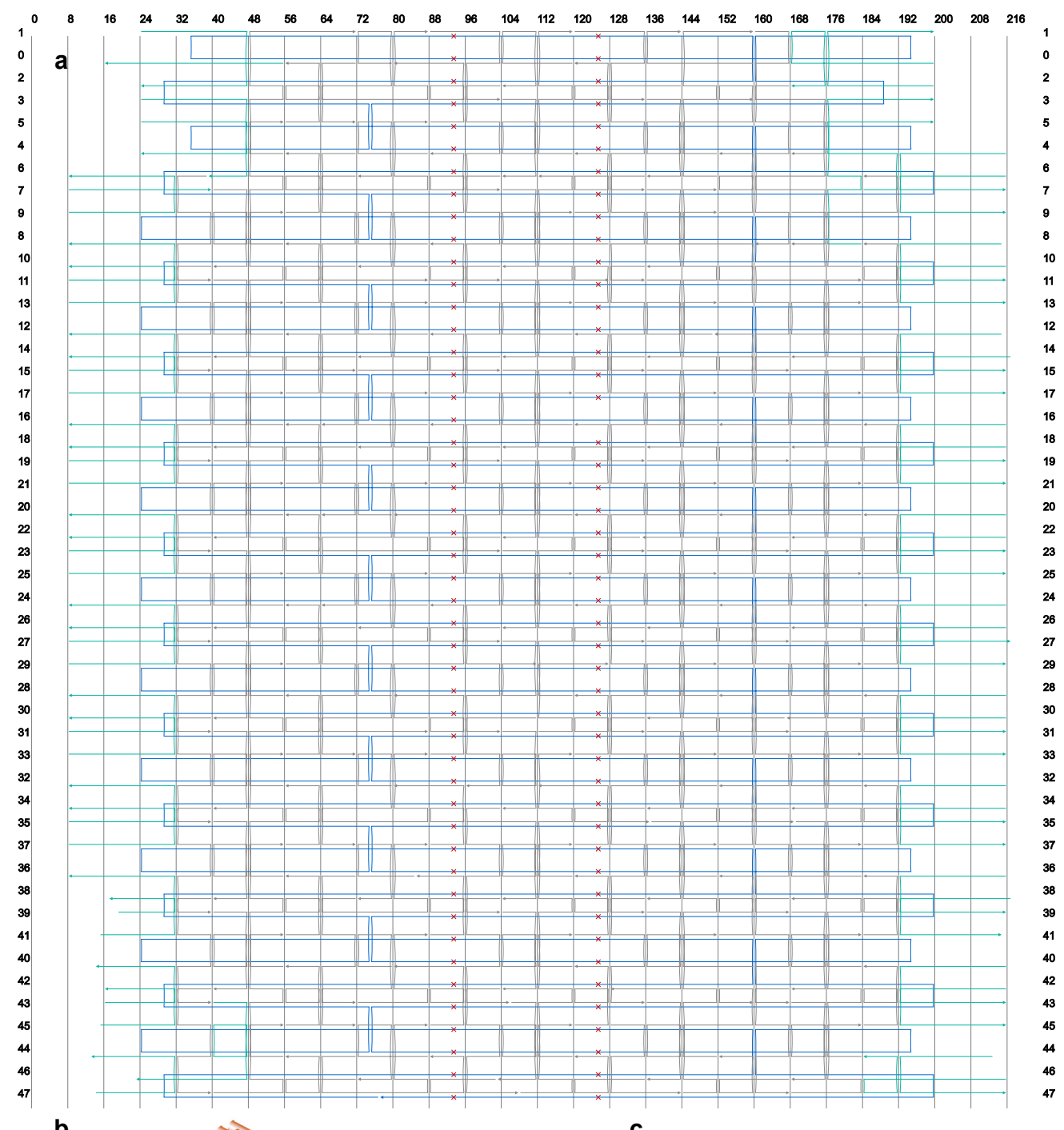

b

C

Figure S1. Design of L. (a) the design blueprint of L in cadnanoSQ. The scaffold DNA is in blue, while DNA staples are in gray. The DNA staples colored in cyan have polyA sequences at their non-hybridized parts, which can help reducing aggregation. (b) 3D view of L. (c) the cross-section view of $\mathrm{L}$ in cadnanoSQ. 


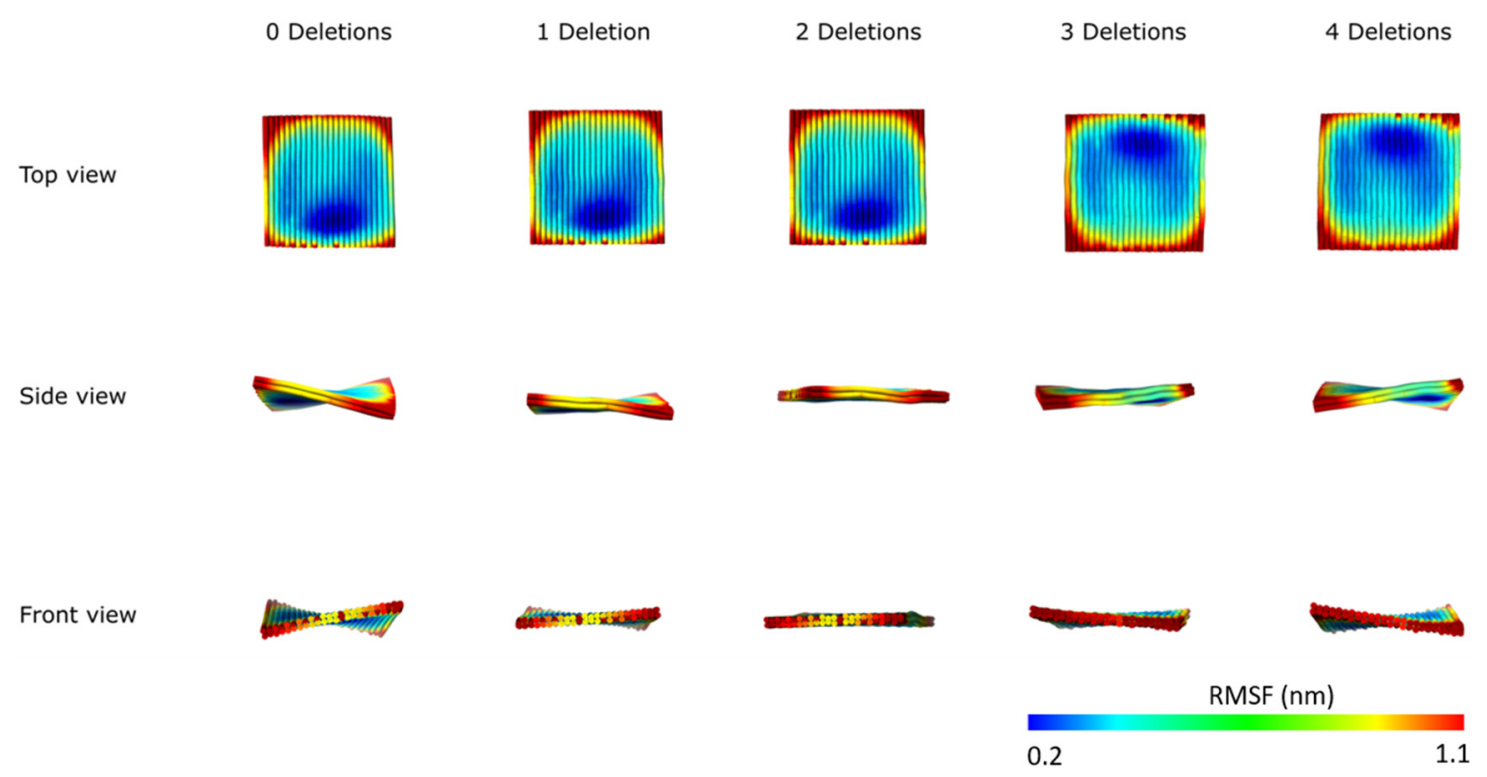

Figure S2. The computational feedback on the 3D structure of different L designs. As shown in Extended Fig. 1, there are 2 columns of deleted bases, where the red " $x$ " marks locate, in the scaffold DNA routing. We chose the number of columns for bases deletions by checking the 3D structure prediction in CanDo (https://cando-dna-origami.org/). The template with 2 columns of base deletions (2 Deletions) shows a relatively flat 3D configuration, while other designs (0 Deletions, 1 Deletions, 3 Deletions and 4 Deletions) present twisted configurations. Thus, we used the template having 2 Deletions for next peptide patterning. 

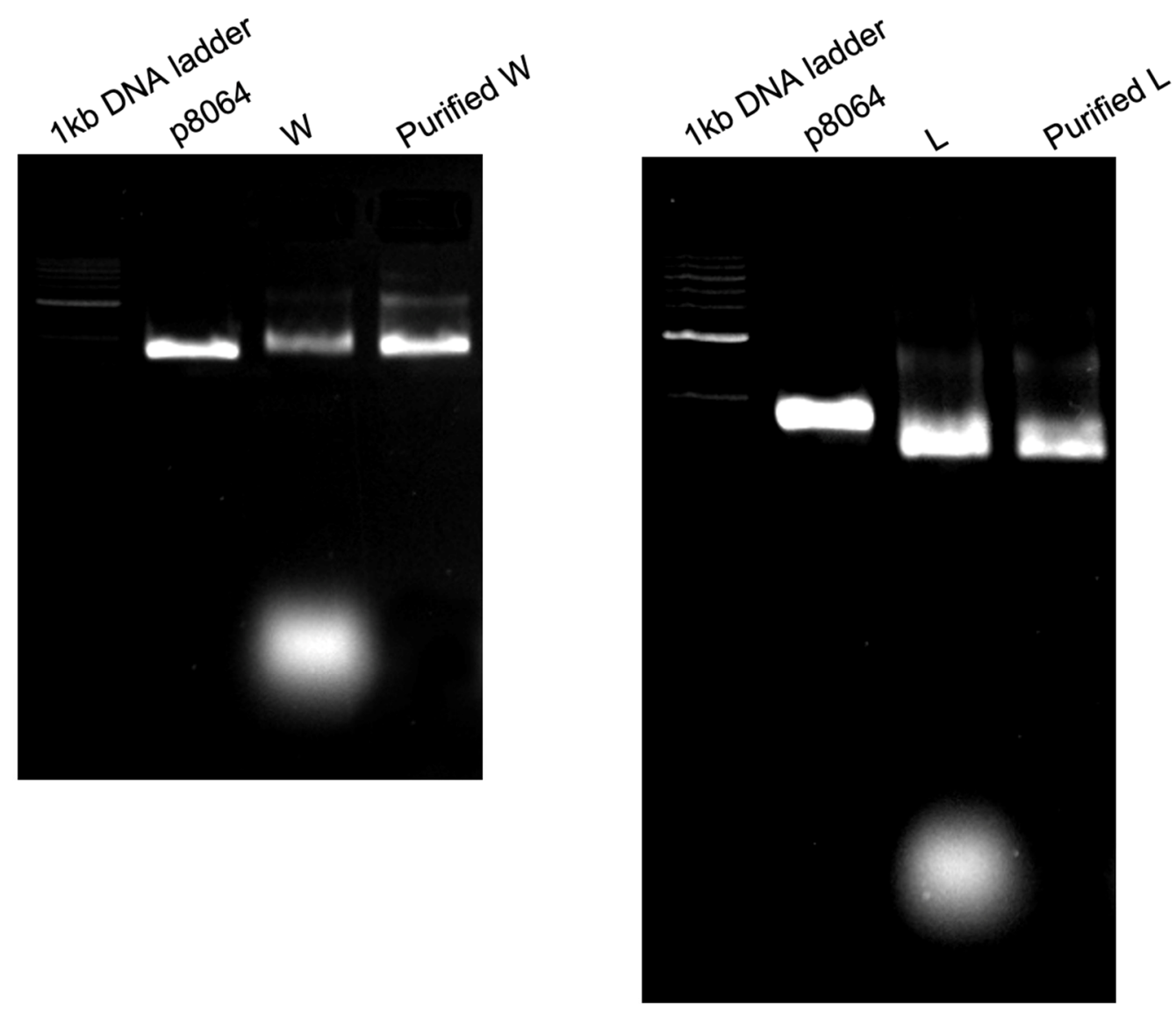

Figure S3. 2\% agarose gel electrophoresis of W (Left) and L (Right), before and after purification. The band (the bottom one) stands for the DNA staples, which were completely washed away by the purification process. 


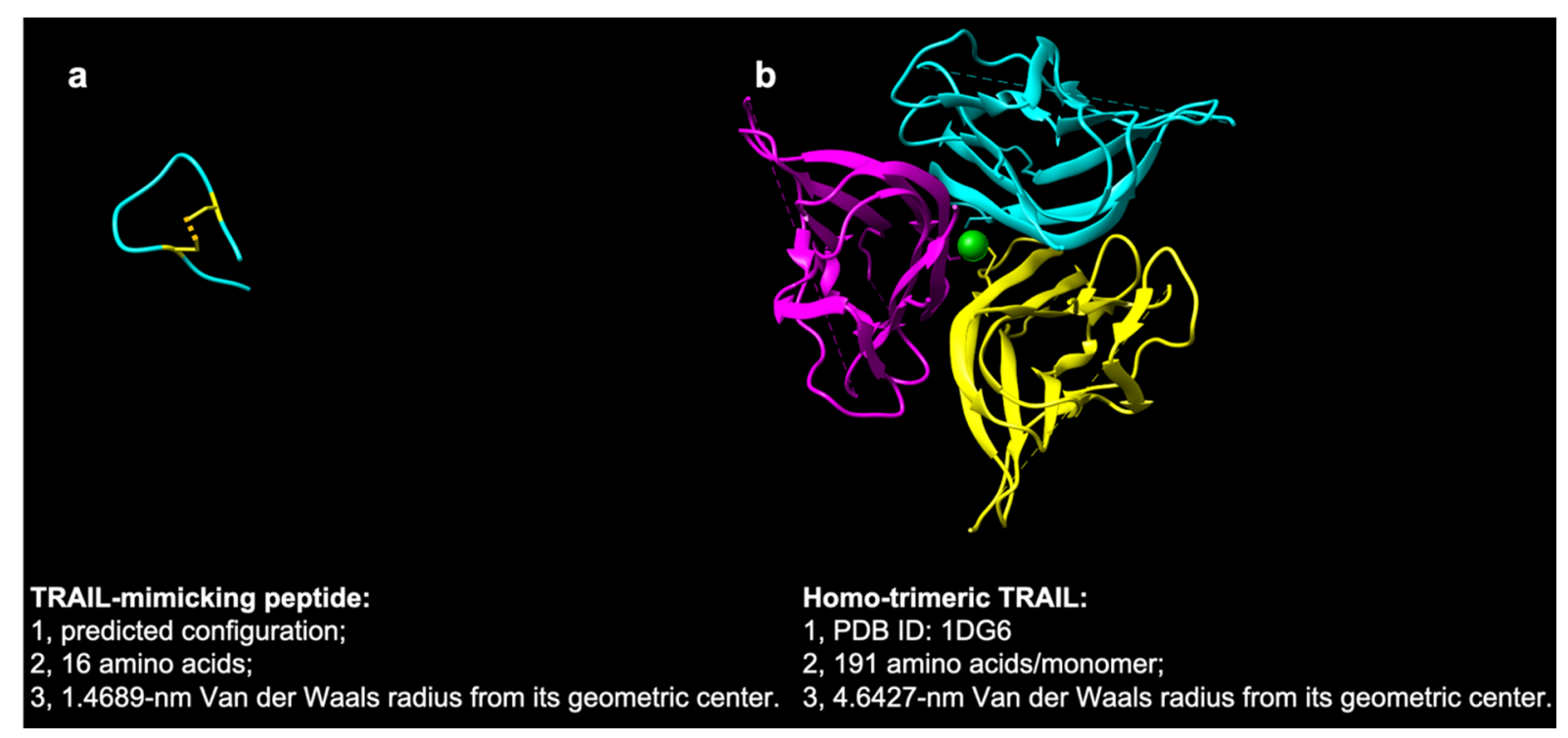

Figure S4. Basic structural information of the peptide and TRAIL trimer. (a) peptide; (b) homotrimeric TRAIL. To predict the spatial structure of the peptide, its sequence (WDCLDNRIGRRQCVKL) was submitted to PEP-FOLD server (https://bioserv.rpbs.univparis-diderot.fr/services/PEP-FOLD/) to compute its PDB coordinates information, which was

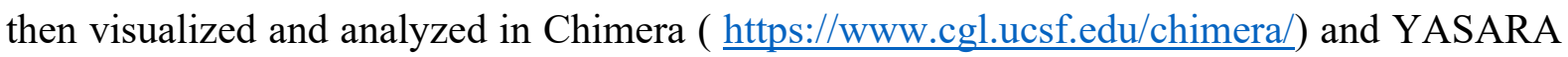
(http://www.yasara.org/). The peptide is cyclized by a disulfide bond between two cysteines. The Human TRAIL trimer structure, with 1DG6 as its PDB ID, was directly sourced from RCSB PDB (https://www.rcsb.org/). Its parameter of Van der Walls radius from its geometric center was also analyzed in YASARA. 

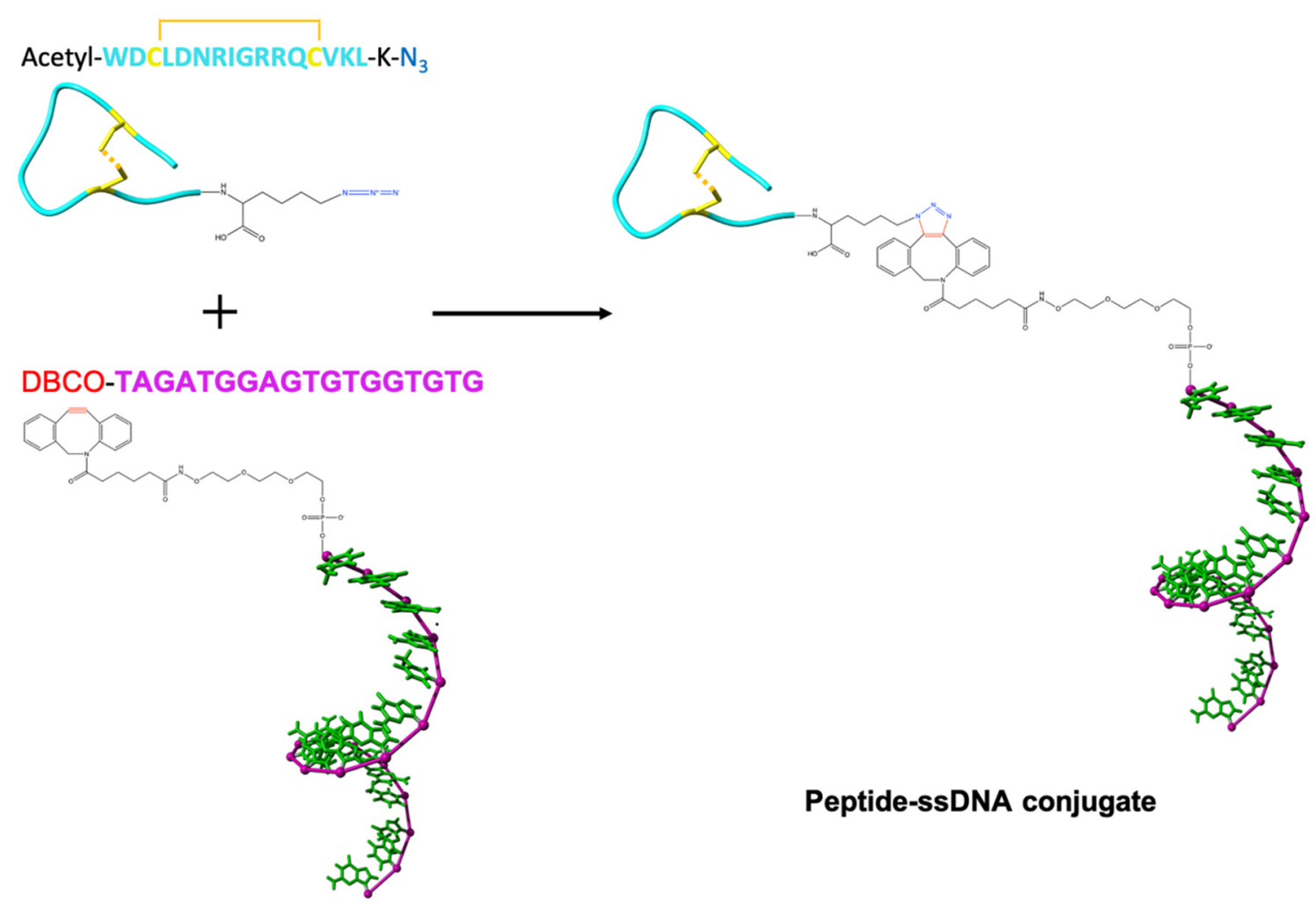

Peptide-ssDNA conjugate

Figure S5. Peptide-DNA conjugation. The peptide is modified with an azide group at its Cterminal; the 18-base long ssDNA is tagged a DBCO at its 5-prime. The reaction is a copper free click chemistry reaction, which has a high reaction efficiency in PBS ( $\mathrm{pH} 7.4$ ), under room temperature. 


\begin{tabular}{|l|l|l|l|l|l|l|l|l|}
\hline Lane & $\mathbf{1}$ & $\mathbf{2}$ & $\mathbf{3}$ & $\mathbf{4}$ & $\mathbf{5}$ & $\mathbf{6}$ & $\mathbf{7}$ & $\mathbf{8}$ \\
\hline Sample & DNA ladder & DBCO-DNA & Peptide & Conjugate & Conjugate + Cy5-comDNA & Cy5-comDNA & Cy5-comDNA + DBCO-DNA & DNA ladder \\
\hline
\end{tabular}

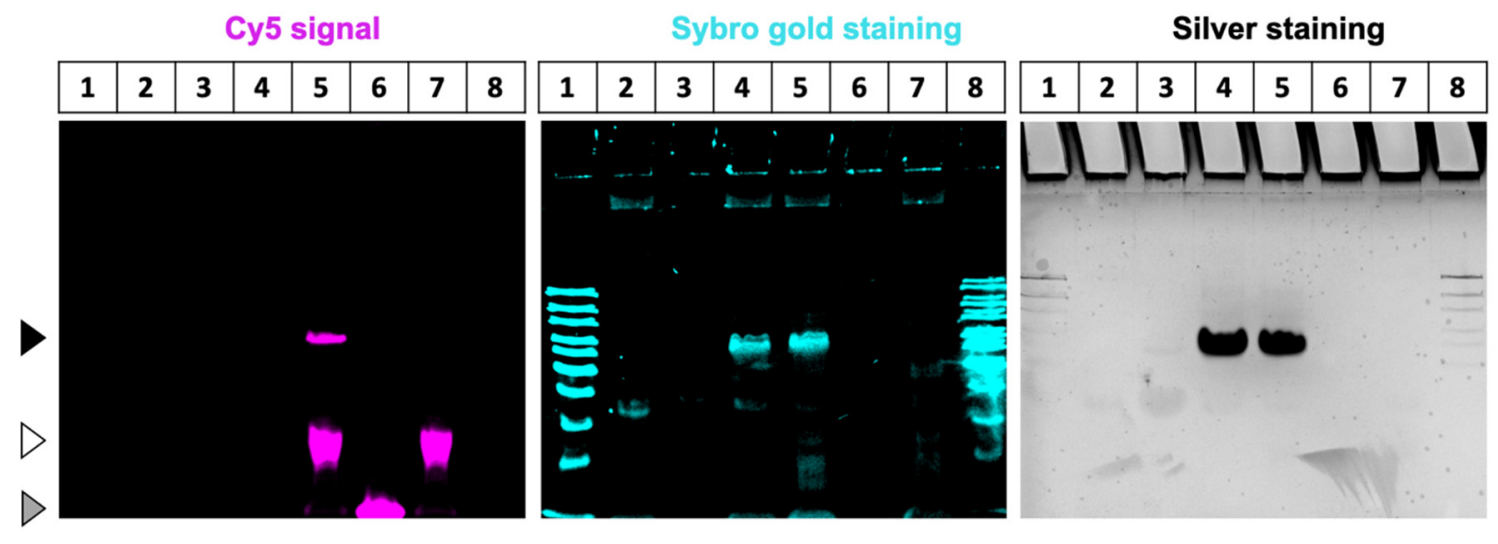

Sybro gold staining

Silver staining

: Conjugate position on the gel

$D$ : Formed double-stranded DNA position on the gel

$D$ : Cy5-comDNA position on the gel

Figure S6. Validation the peptide-DNA conjugation by gel followed by silver staining. 18\% native polyacrylamide gel was used. We used a Cy5-labled complementary DNA (Cy5comDNA) to hybridize the DNA of the conjugate, thus we can visualize the conjugate under Cy5 channel. All of the samples are indicated as in the figure. The gel was imaged under Cy5 channel; nucleic acids on the gel was stained by SYBR gold; peptides were stained by silver staining. 


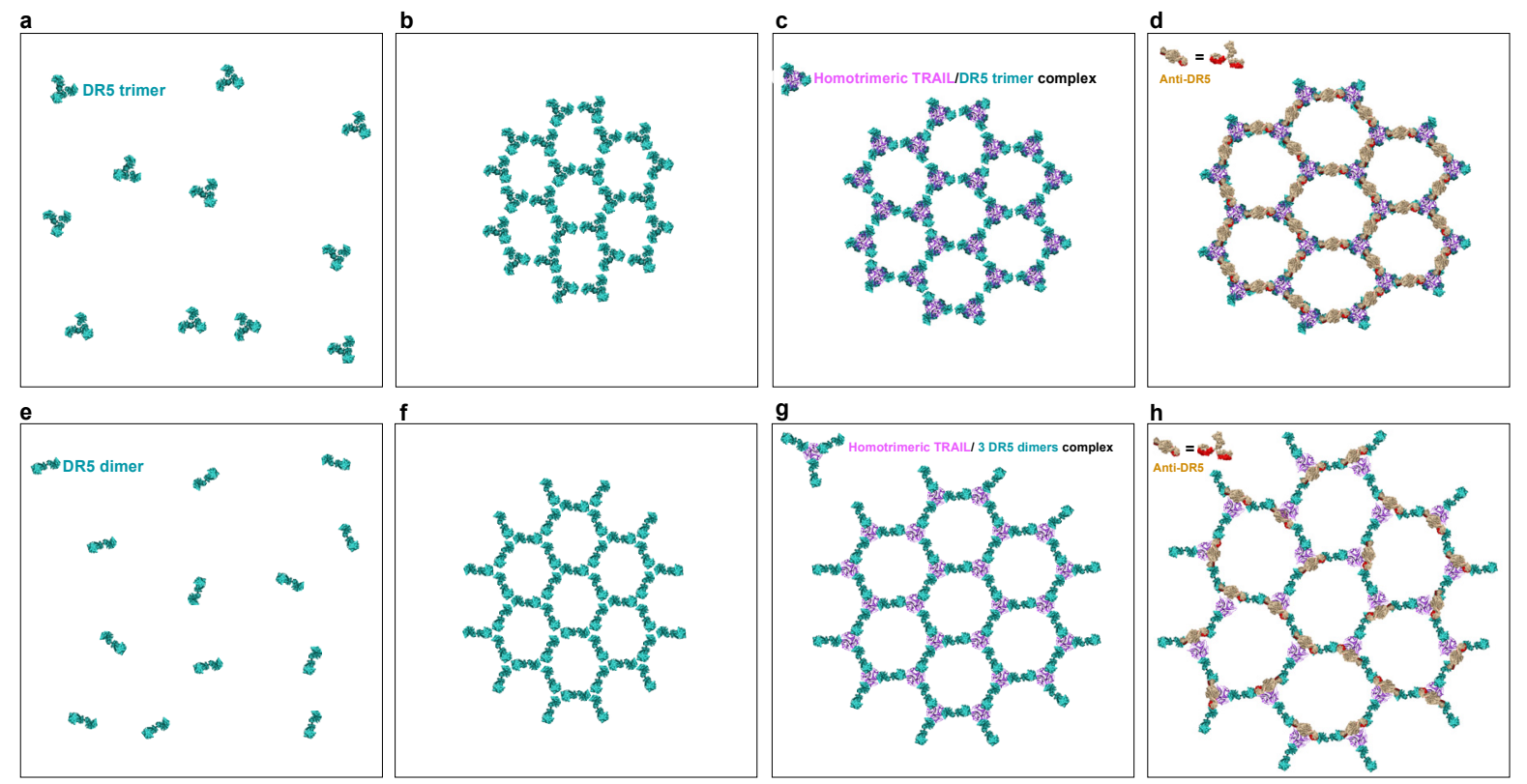

Figure S7. Current interpretation of apoptosis-driving hexagonal DR5 networks (top view from the outside of a cell surface). (a) Surface representations of pre-ligand DR5 trimers. (b) The proposed active hexagonal DR5 network via dimerization of DR5 trimers. (c) The proposed active hexagonal network of homo-trimeric TRAIL/DR5 trimer complexes. (d) The hexagonal homo-trimeric TRAIL/DR5 trimer network promoted and maintained by the antibody AMG 655. (e) Surface representations of pre-ligand DR5 dimers. (f) The proposed active hexagonal DR5 network via trimerization of DR5 dimers. (g) The proposed active hexagonal network of homo-trimeric TRAIL/DR5 dimer complexes. (h) The hexagonal homo-trimeric TRAIL/DR5 dimer network promoted and maintained by antibody AMG 655 . 


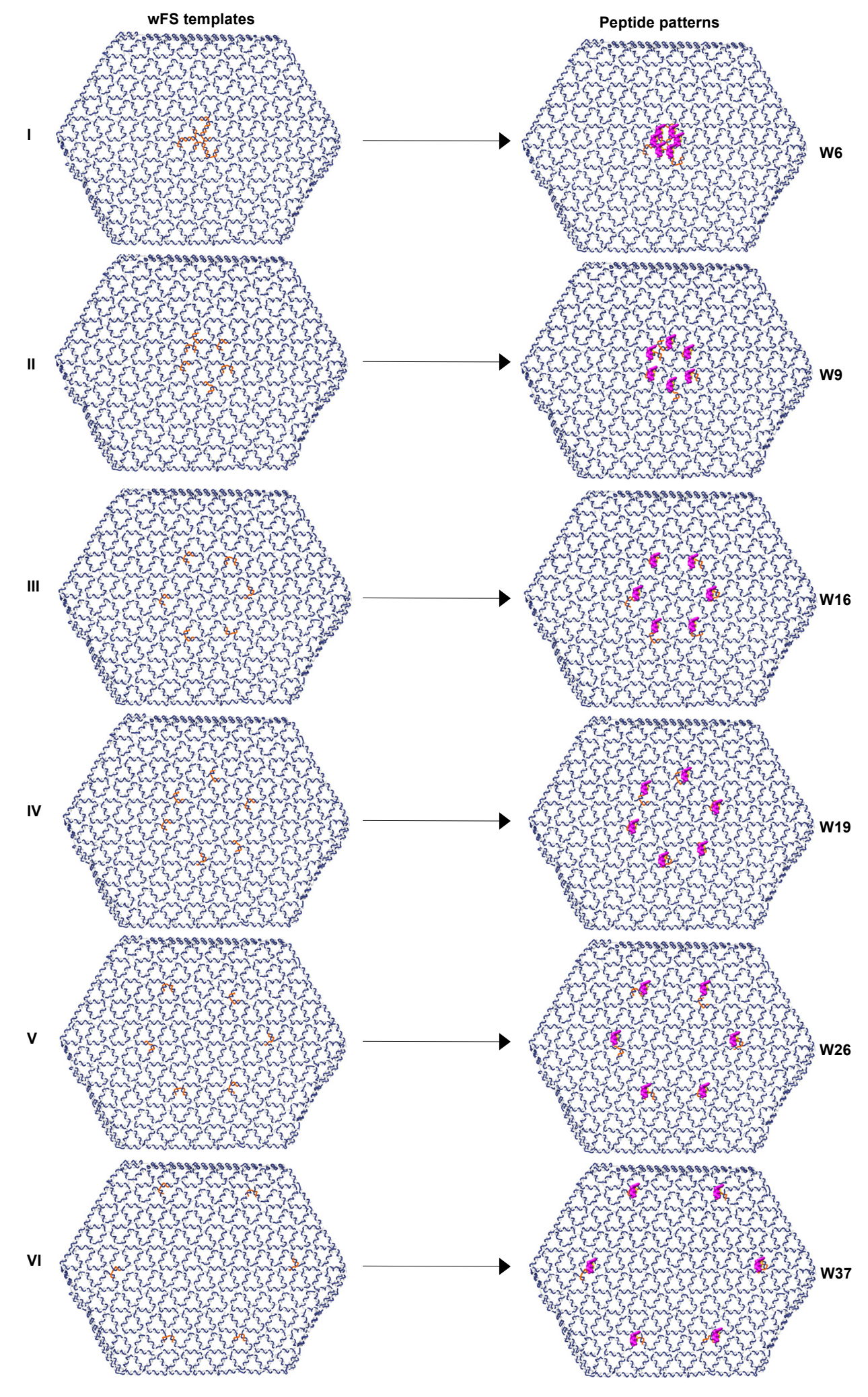

Figure S8. Designs of peptide patterns on W. The sizes of hexagonal patterns are decided by the choices of specific DNA staples on W. These staples were colored in orange, while blue is the scaffold DNA and gray means other DNA staples. These staples in orange contain protruding single-stranded DNA at their 5 primes, thus they can hybridize with the DNApeptide conjugate. 


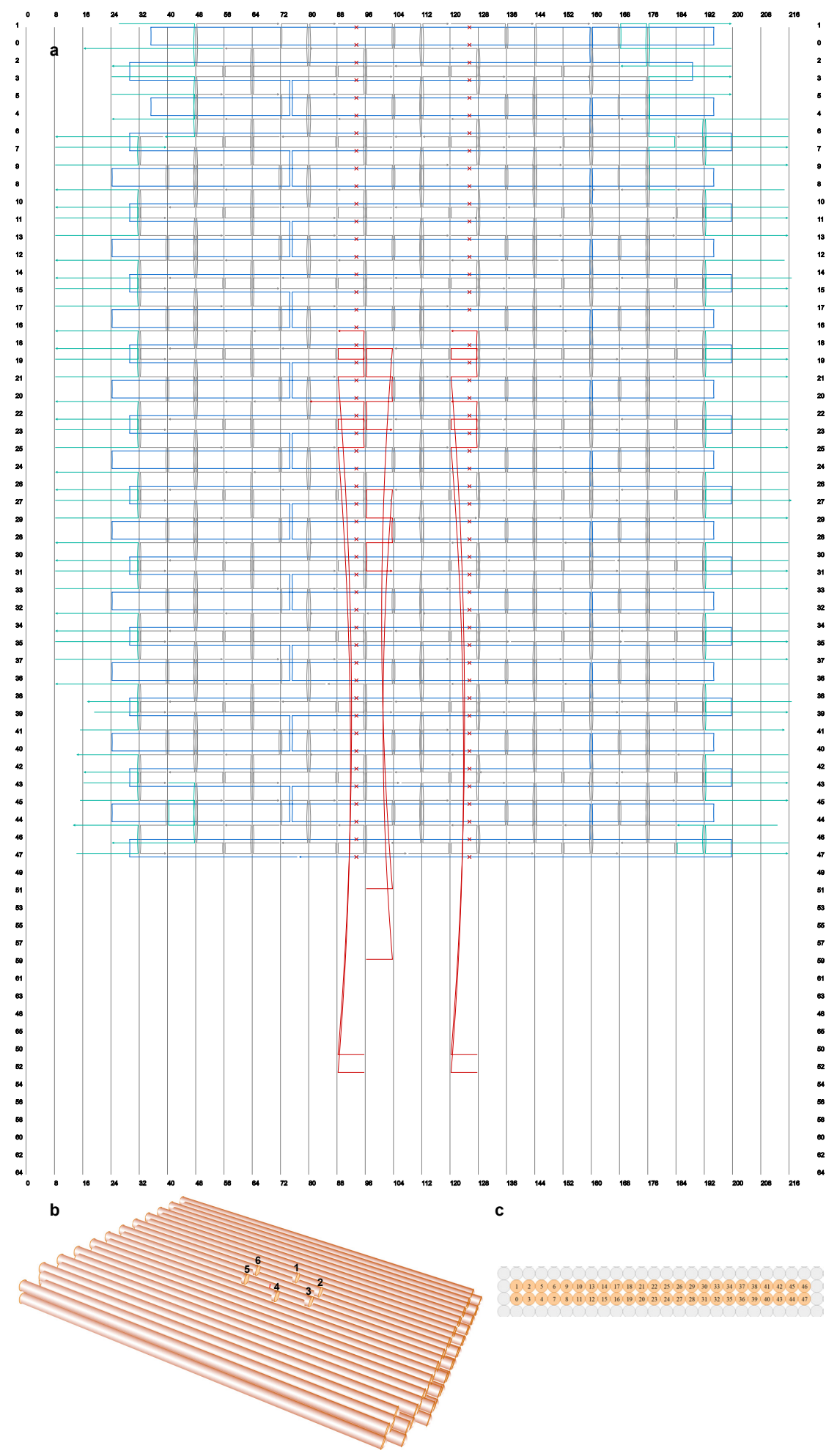

Figure S9. Design of the template for L6. a, the design blueprint of the template in cadnanoSQ. The scaffold DNA is in blue, while DNA staples are in gray. The DNA staples colored in cyan have polyA sequences at their non-hybridized parts, which can help reducing aggregation. The 6 staples having protruding sequences at their 5 primes, for the docking of DNA-peptide conjugate, were colored in red. $\mathrm{b}$, the $3 \mathrm{D}$ view of the tempalte, showing that the 6 protruding sites are presenting a hexagonal pattern, and the distance between adjacent sites are around 5 nm. c, the cross-section view of the template in cadnanoSQ. 


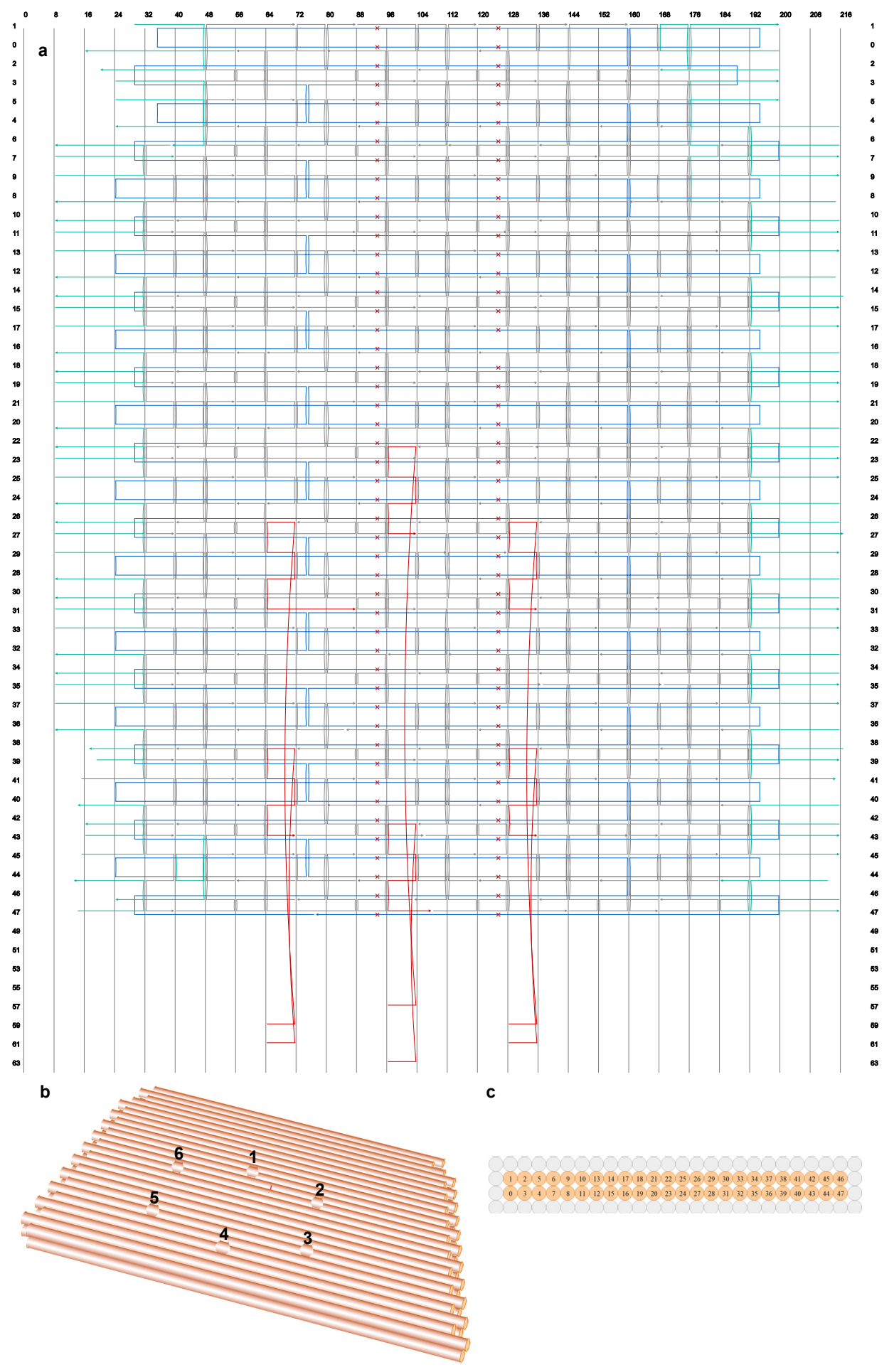

Figure S10. Design of the template for L11. (a) the design blueprint of the template in cadnanoSQ. The scaffold DNA is in blue, while DNA staples are in gray. The DNA staples colored in cyan have polyA sequences at their non-hybridized parts, which can help reducing aggregation. The 6 staples having protruding sequences at their 5 primes, for the docking of DNA-peptide conjugate, were colored in red. (b) the $3 \mathrm{D}$ view of the tempalte, showing that the 6 protruding sites are presenting a hexagonal pattern, and the distance between adjacent sites are around $10 \mathrm{~nm}$. (c) the cross-section view of the template in cadnanoSQ. 

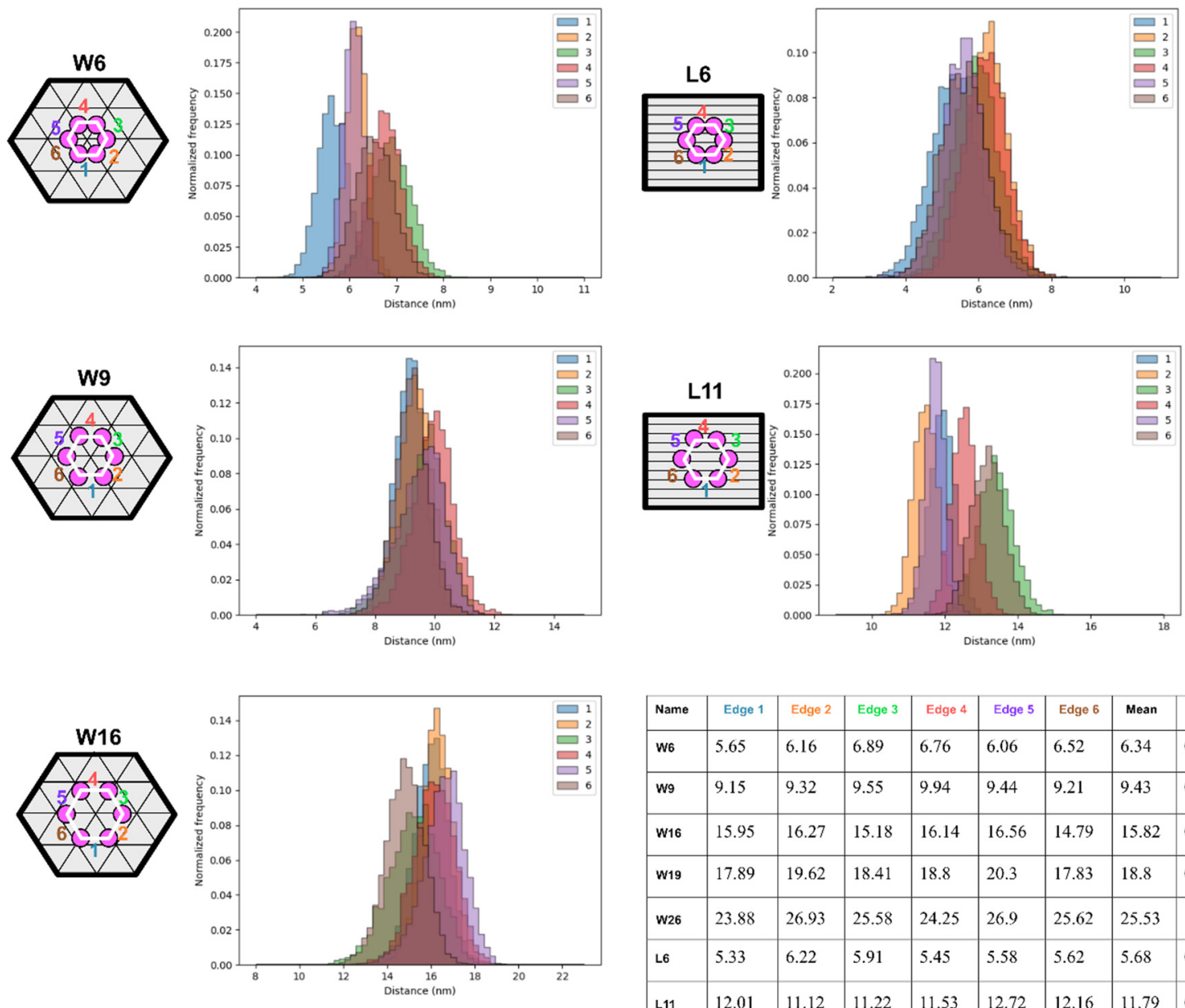

\begin{tabular}{|l|l|l|l|l|l|l|l|l|}
\hline Name & Edge 1 & Edge 2 & Edge 3 & Edge 4 & Edge 5 & Edge 6 & Mean & SD \\
\hline W6 & 5.65 & 6.16 & 6.89 & 6.76 & 6.06 & 6.52 & 6.34 & 0.46 \\
\hline W9 & 9.15 & 9.32 & 9.55 & 9.94 & 9.44 & 9.21 & 9.43 & 0.95 \\
\hline W16 & 15.95 & 16.27 & 15.18 & 16.14 & 16.56 & 14.79 & 15.82 & 0.68 \\
\hline W19 & 17.89 & 19.62 & 18.41 & 18.8 & 20.3 & 17.83 & 18.8 & 0.98 \\
\hline W26 & 23.88 & 26.93 & 25.58 & 24.25 & 26.9 & 25.62 & 25.53 & 1.28 \\
\hline L6 & 5.33 & 6.22 & 5.91 & 5.45 & 5.58 & 5.62 & 5.68 & 0.32 \\
\hline L11 & 12.01 & 11.12 & 11.22 & 11.53 & 12.72 & 12.16 & 11.79 & 0.28 \\
\hline
\end{tabular}
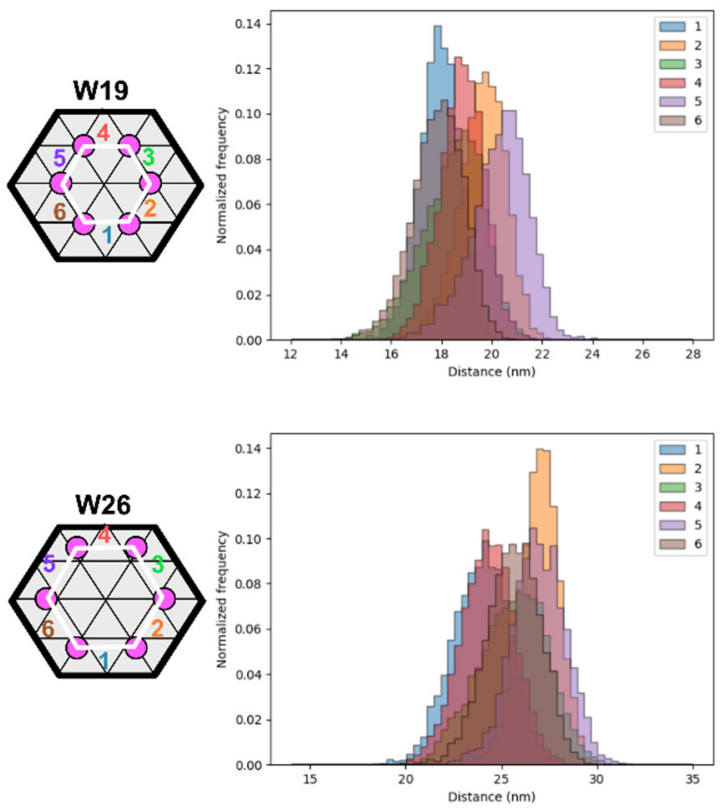

Figure S11. Nucleotide-to-nucleotide distance measurement through oxDNA molecular dynamics simulation. For each hexagonal pattern on DNA origami, the distance distribution (within the oxDNA simulation time frame) of each edge is shown in the distribution histograms. 
The mean distance of each edge, the mean edge distance of a hexagonal pattern and corresponding standard deviation (SD) are listed in the table.

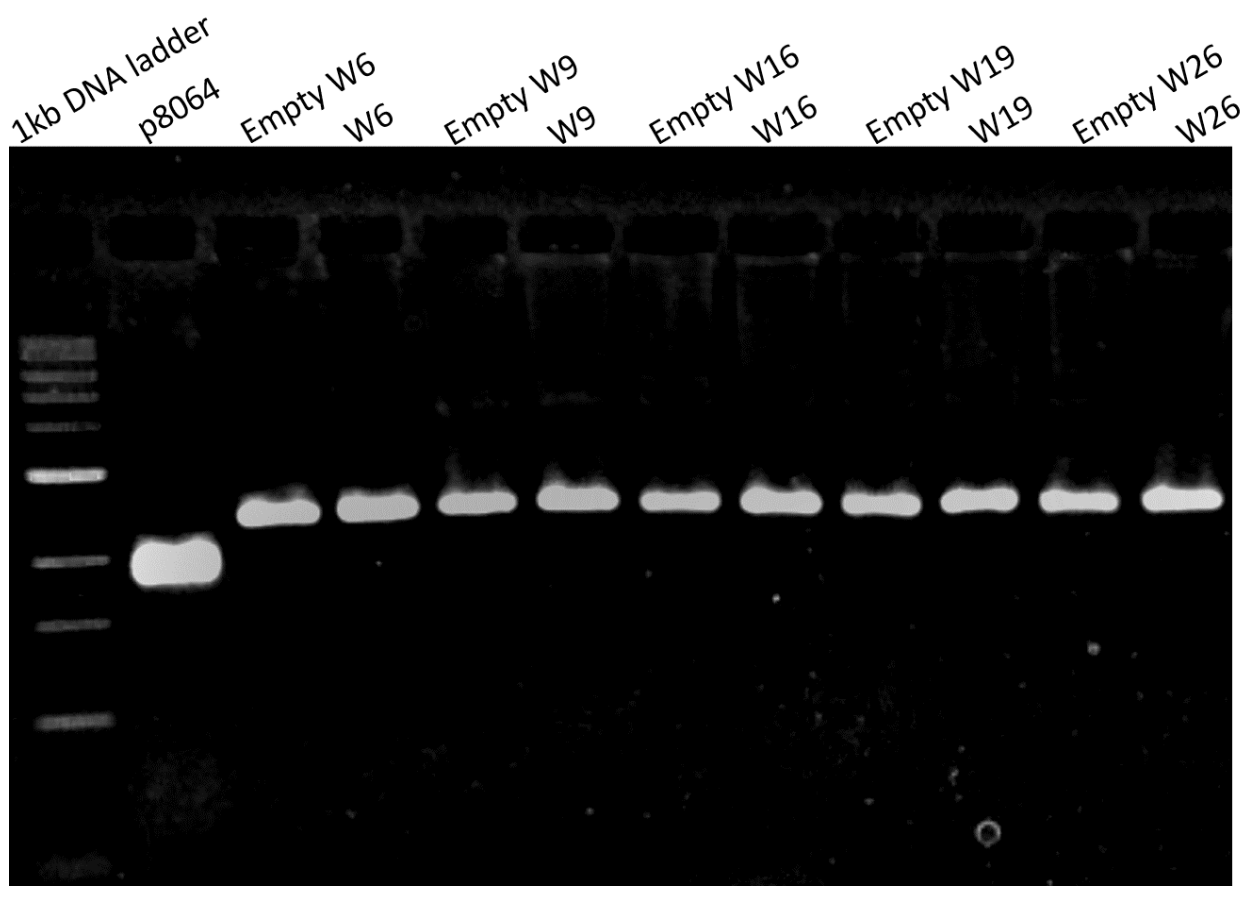

Figure S12. 2\% agarose gel electrophoresis of all W (with and without peptide attachment) structures. The sample of each well is indicated as in the figure.

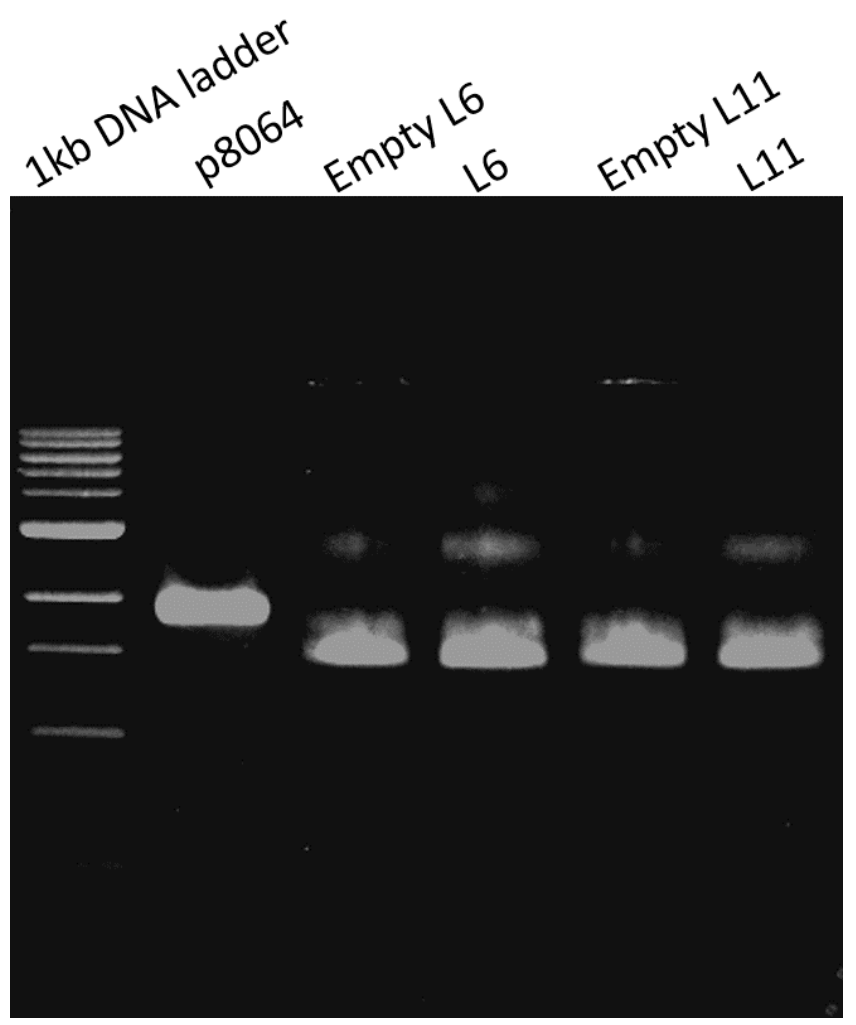


Figure S13. 2\% agarose gel electrophoresis of all L (with and without peptide attachment) structures. The sample of each well is indicated as in the figure.

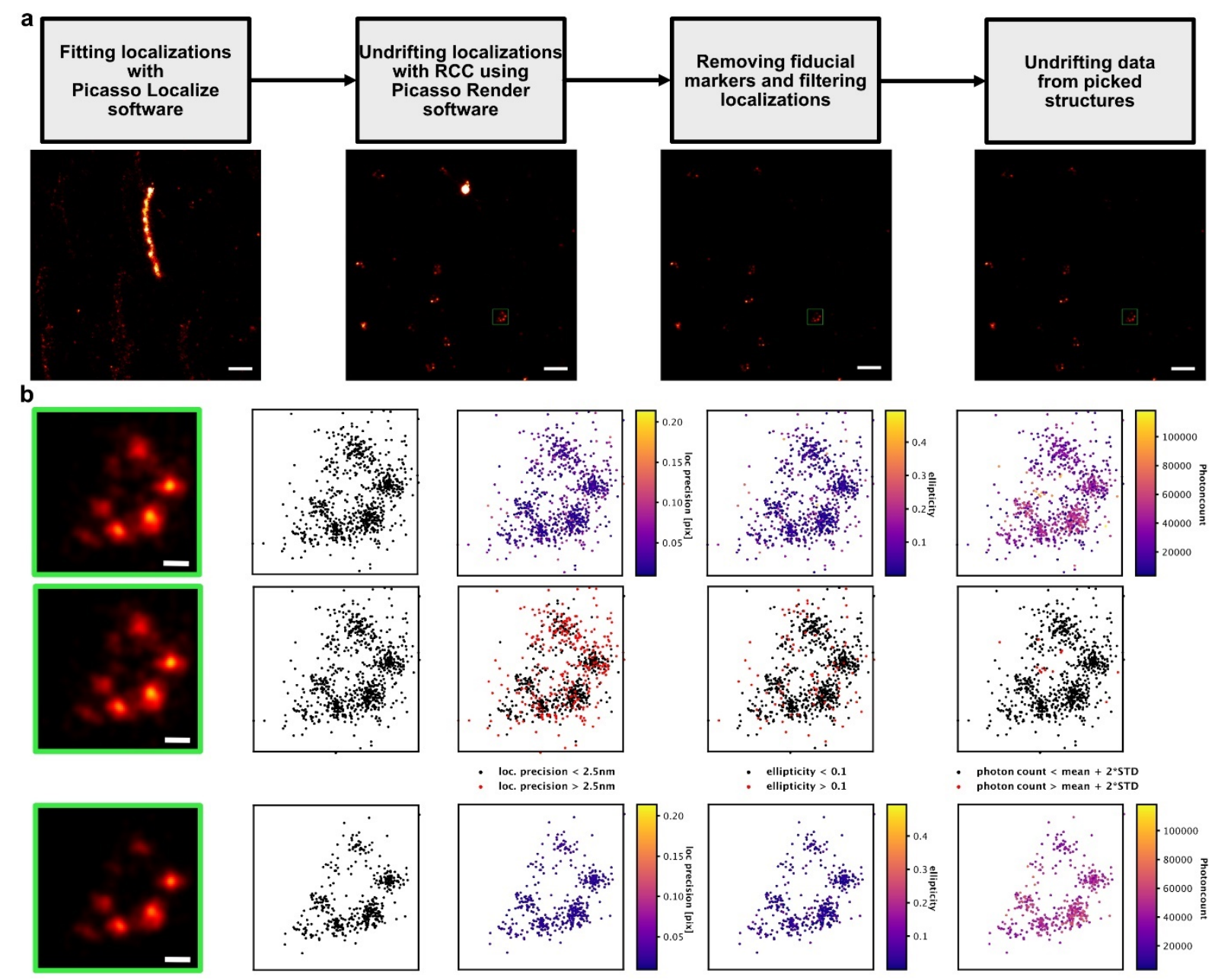

Figure S14. Pre-processing of DNA-PAINT data. (a) Steps of preprocessing the DNA PAINT data with super resolved field of view images of the resulting DNA PAINT localization data of the TRAIL-peptide functionalized wFS37 structures (scale bar=200nm). (b) Plots of localizations belonging to a TRAIL-peptide functionalized wFS37 structure taken from the FOV image (green rectangle) showing localization properties before filtering (upper row), localizations removed by the corresponding filters (red) (middle row) and localization properties after filtering (bottom row) along the super resolution image rendered from the corresponding localizations (scale bar $=20 \mathrm{~nm})$. 

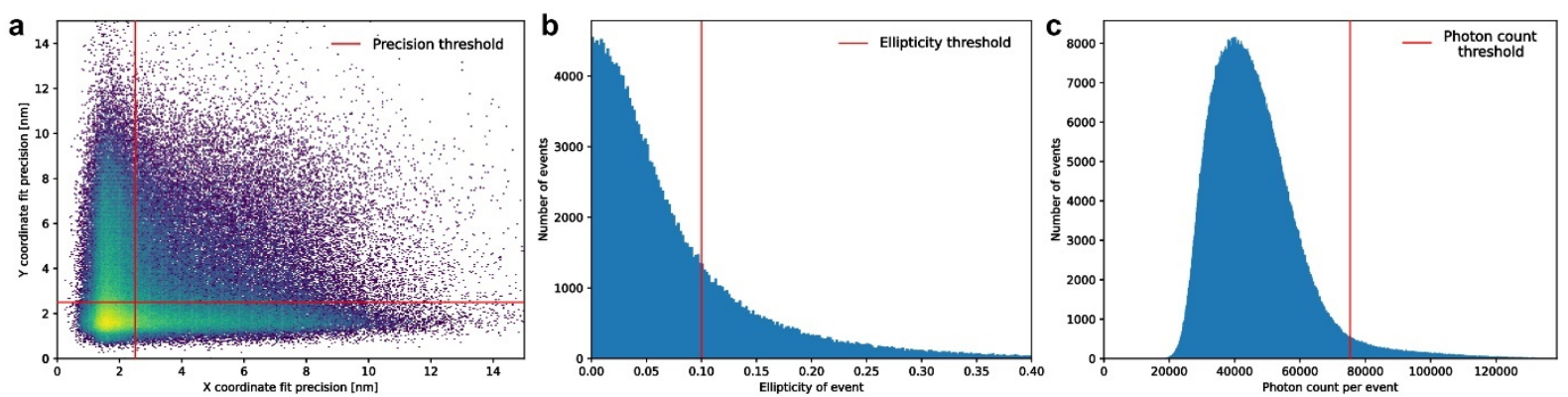

Figure S15. Plots of localization properties and their respective cutoffs used for filtering. (a) Plots of localization fitting precision, (b) ellipticity, (c) and photon counts of the localization data produced by imaging TRAIL-peptide functionalized wFS37 structure with the thresholds applied during preprocessing indicated. 

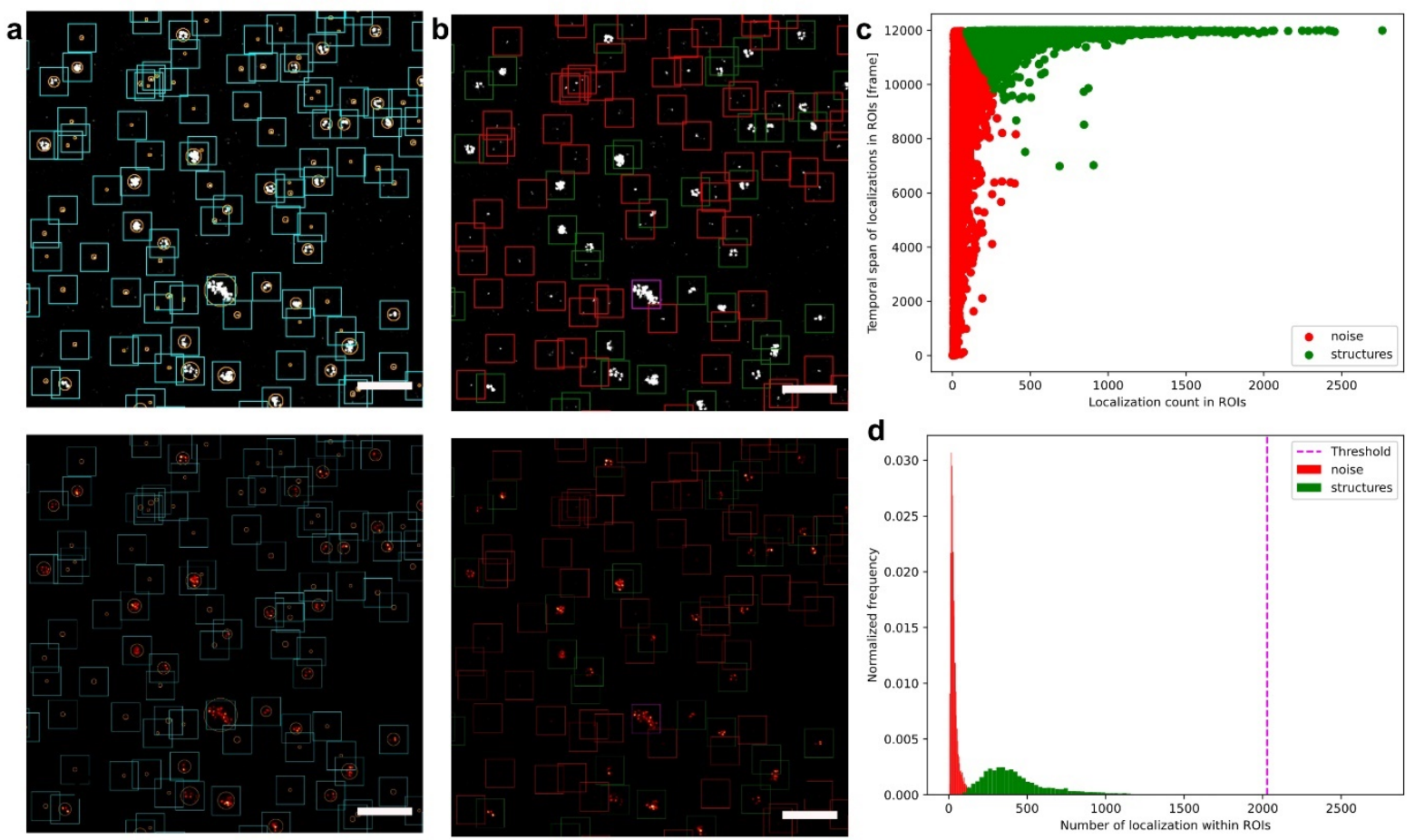

Figure S16. Detection of DNA origami ROI coordinates from DNA-PAINT data (A-D) Steps for DNA origami structure position detection in DNA PAINT data presented on the gray scale (top row) and colormap FOV images (bottom row) of W37 structures (scalebars $=500 \mathrm{~nm}$ ). (a) Possible positions of origamis structures were found by detecting contours (orange circles) in the grayscale low resolution images rendered from the data and uniform origami ROI coordinates (cyan boxes) were calculated using the centroid coordinates of the contours. (b) The origami ROIs containing noise (red boxes) were filtered out based on the number and temporal span of localization in them, ROIs containing aggregates of structures (magenta boxes) were filtered out by removing ROIs containing 10 STD more localization than the mean of non-noise containing ROIs, the remaining ROIs (green boxes) were kept as origami ROIs for further processing. (c) Scatter plot of ROI localization numbers and temporal spans clustered into 2 groups using k-means clustering on two-component PCA transformed data resulting in a cluster of ROIs with shorter temporal span containing noise (red) and ROIs containing signal from structures. (d) Distribution plot of localization numbers of clustered ROIs showing the 
localization number threshold (magenta dashed line) above which ROIs containing aggregates of structures were filtered out.

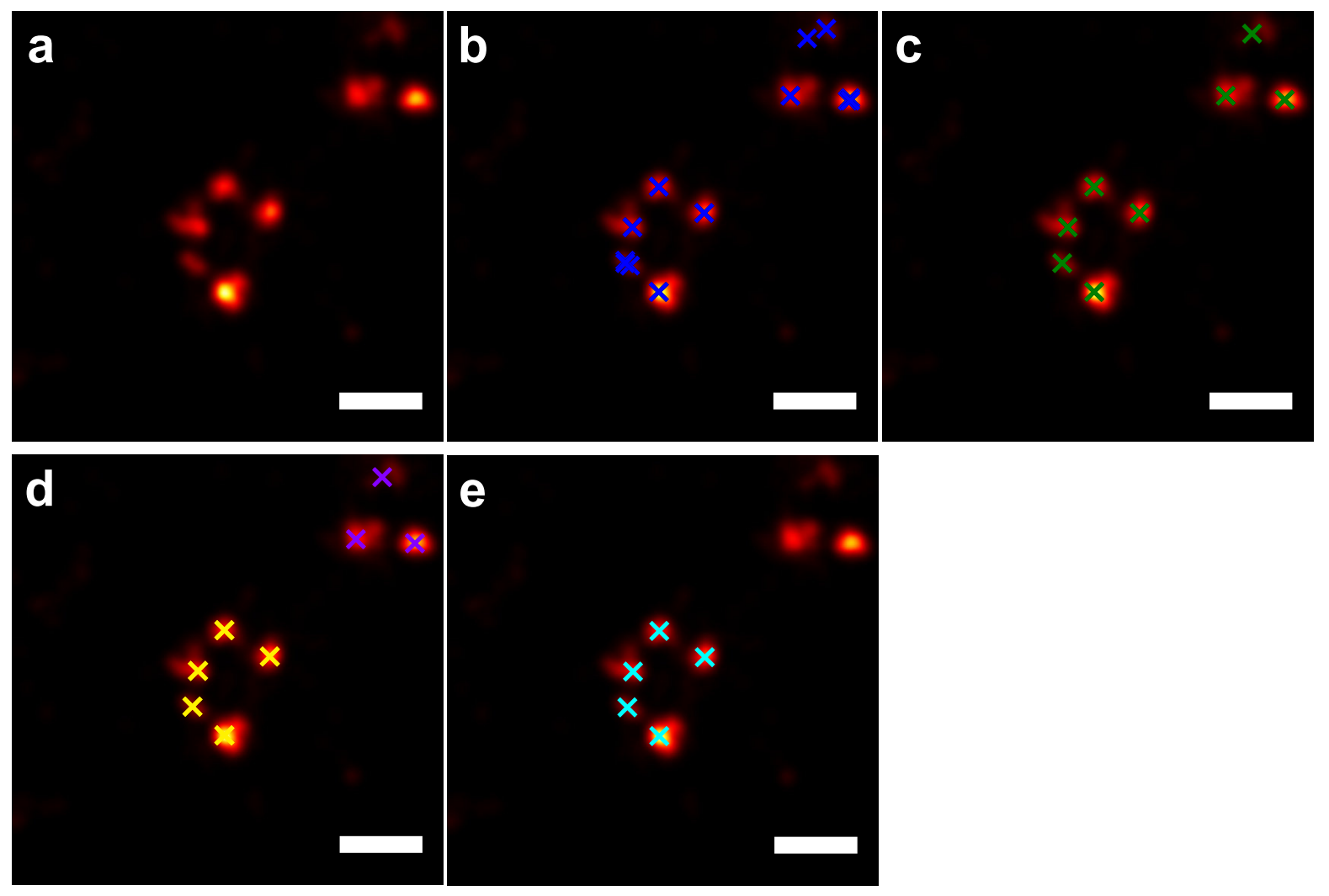

Figure S17. Detection of TRAIL peptides in origami ROIs extracted from DNA-PAINT data. (a) Localizations lying within detected origami ROIs were grouped and rendered. (b) Local maxima (blue crosses) were detected in the rendered image. (c) Maxima lying closer to each other than a distance threshold ( 0.45 site distance) were merged and an average position for them were calculated yielding the estimated position of TRAIL peptides in the ROI (green crosses). (d) TRAIL peptides belonging to individual origamis were grouped together (purple and yellow crosses) via hierarchical clustering with the maximum intra cluster distance set to 2.5 site distance. (e) Finally the cluster with the most number of TRAIL peptides closest to the center of the origami ROI is kept and used for quantification. (Scale bars $=50 \mathrm{~nm}$ ). 


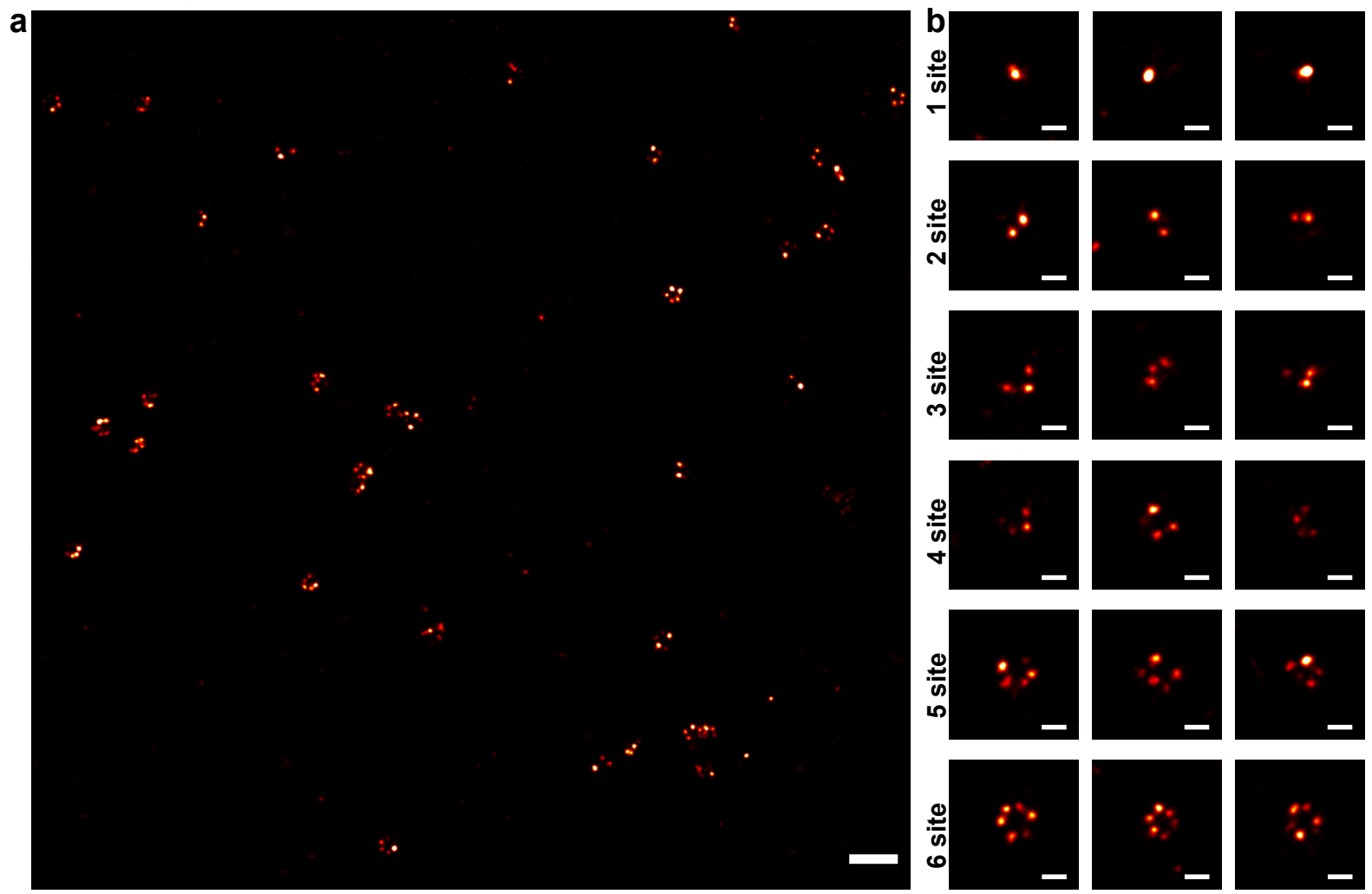

Figure S18. DNA-PAINT images of W37 structures. (a) A field of view DNA PAINT image of the W37 structures (Scale bar $=200 \mathrm{~nm}$ ). (b) Rendered images of origami ROIs containing 1, 2, 3, 4, 5 and 6 detected TRAIL peptides $($ Scale bar $=50 \mathrm{~nm})$. 


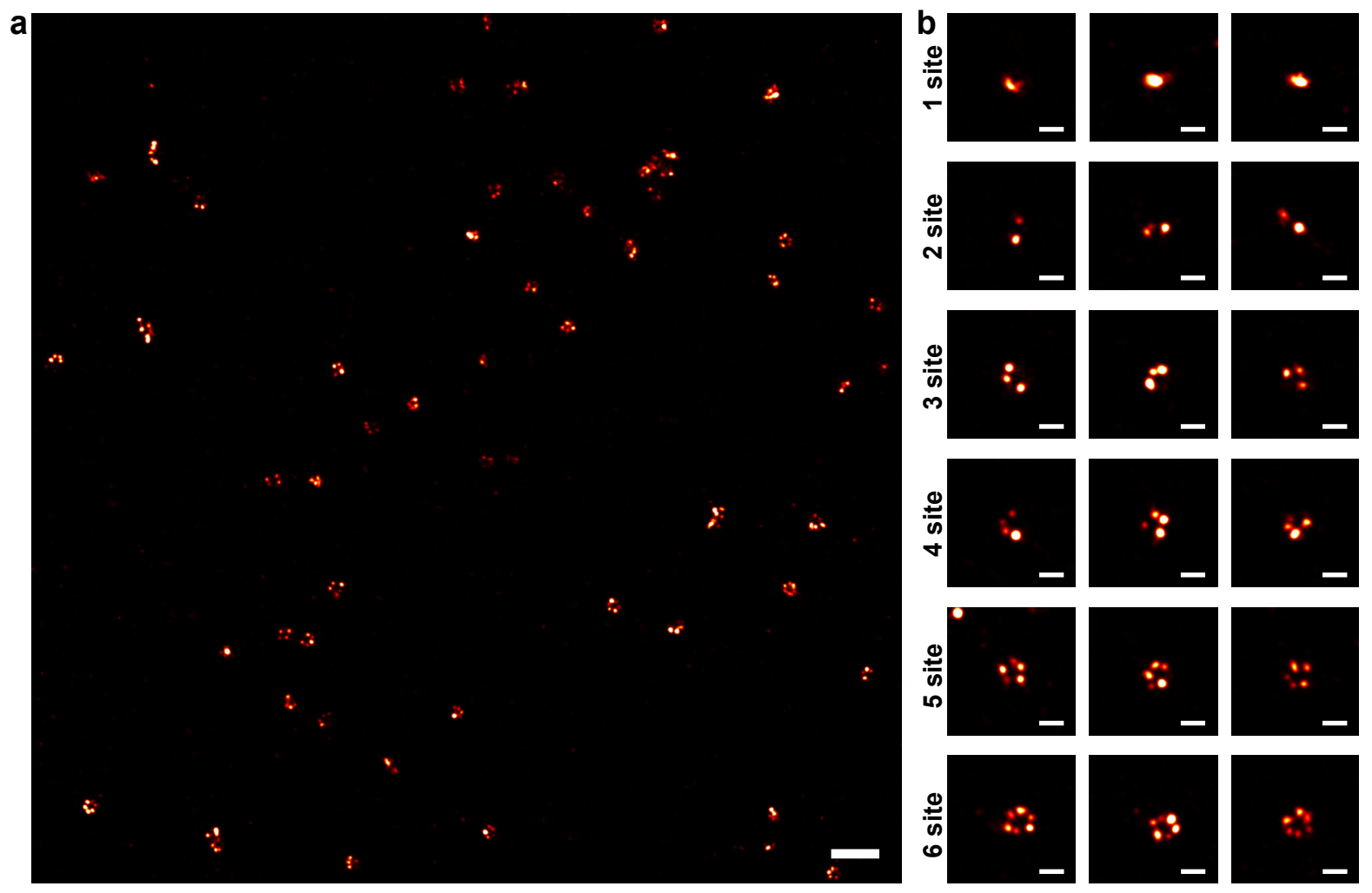

Figure S19. DNA-PAINT images of W28 structures. (a) A field of view DNA PAINT image of the W28 structures (Scale bar $=200 \mathrm{~nm}$ ). (b) Rendered images of origami ROIs containing 1, 2, 3, 4, 5 and 6 detected TRAIL peptides $($ Scale bar $=50 \mathrm{~nm})$. 

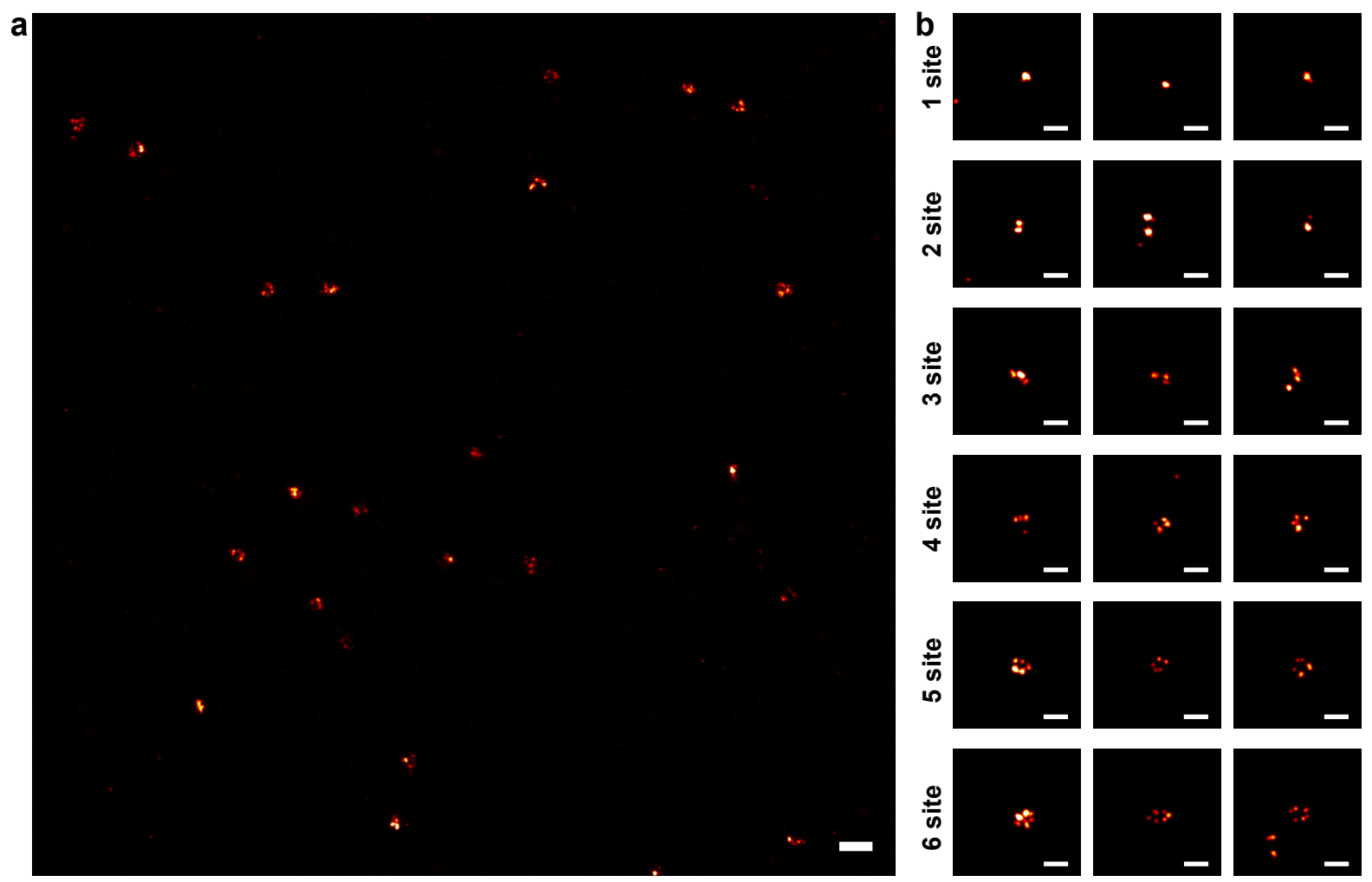

Figure S20. DNA-PAINT images of W19 structures. (a) A field of view DNA PAINT image of the W19 structures (Scale bar $=200 \mathrm{~nm})$. (b) Rendered images of origami ROIs containing 1, 2, 3, 4, 5 and 6 detected TRAIL peptides (Scale bar $=50 \mathrm{~nm})$. 


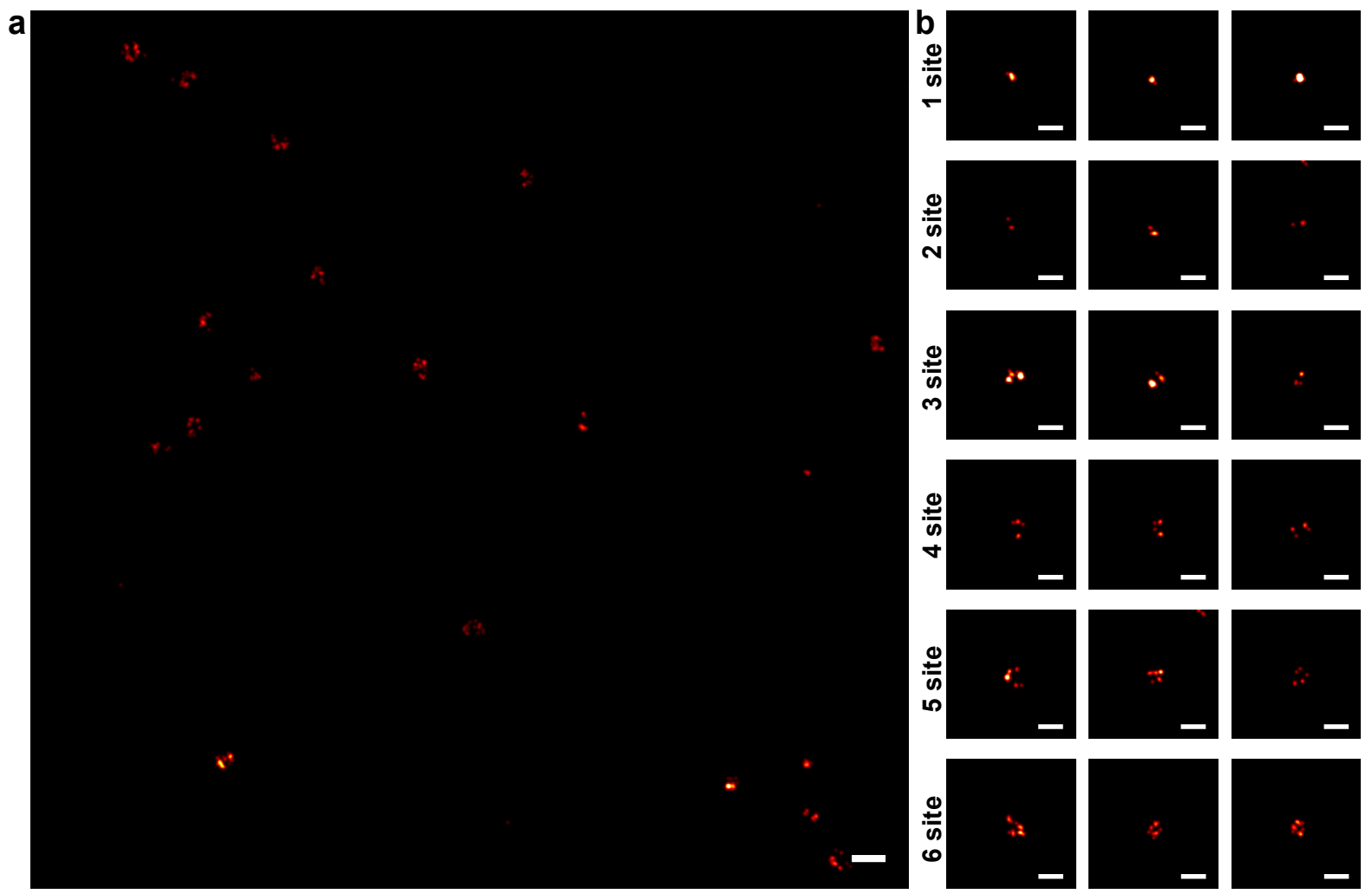

Figure S21. DNA-PAINT images of W16 structures. (a) A field of view DNA PAINT image of the W16 structures (Scale bar $=200 \mathrm{~nm})$. (b) Rendered images of origami ROIs containing 1, 2, 3, 4, 5 and 6 detected TRAIL peptides $($ Scale bar $=50 \mathrm{~nm})$. 


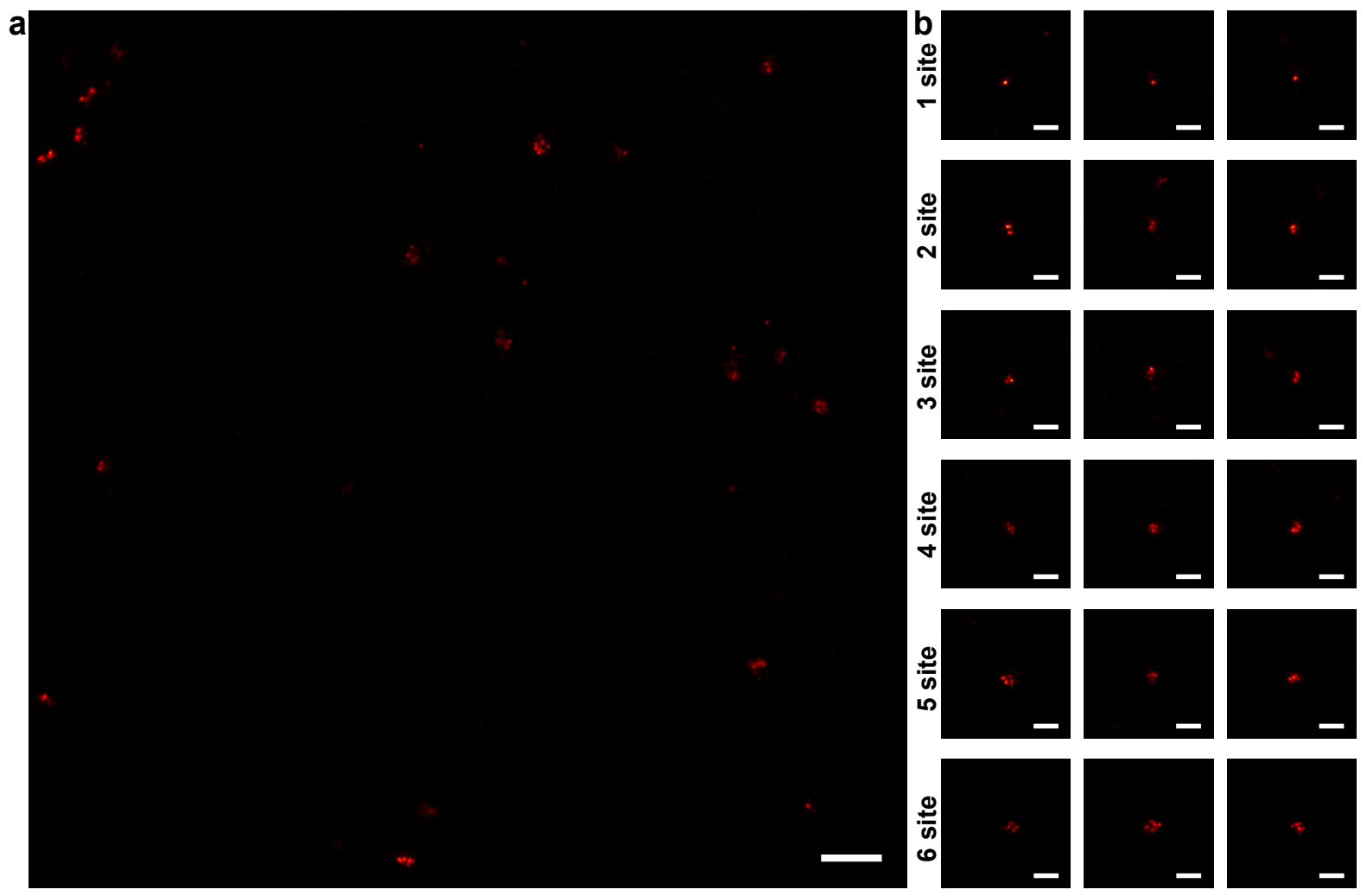

Figure S22| DNA-PAINT images of W9 structures. (a) A field of view DNA PAINT image of the W9 structures (Scale bar $=200 \mathrm{~nm})$. (b) Rendered images of origami ROIs containing 1, 2, 3, 4, 5 and 6 detected TRAIL peptides $($ Scale bar $=50 \mathrm{~nm})$. 


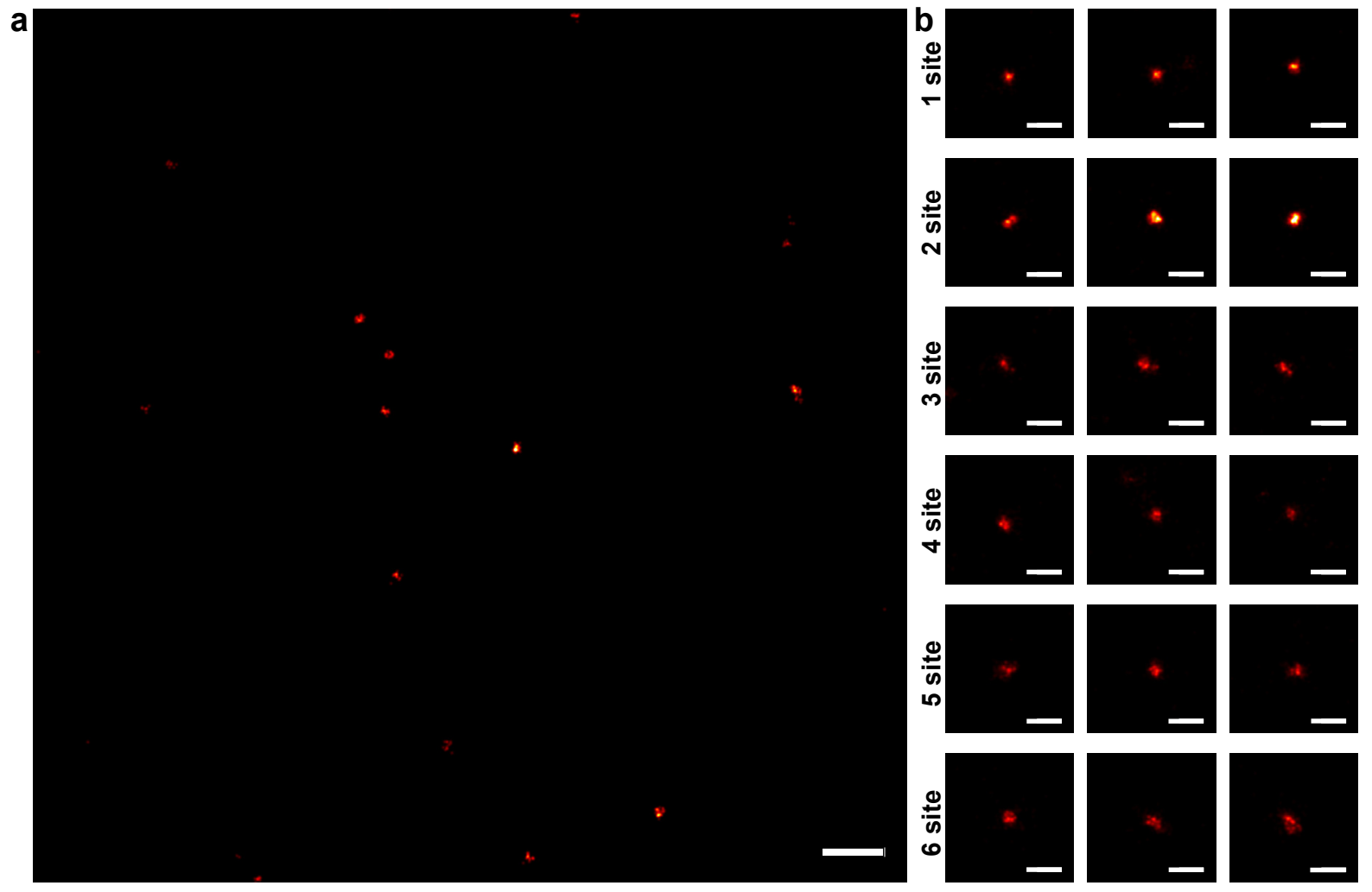

Figure S23. DNA-PAINT images of W6 structures. (a) A field of view DNA PAINT image of the W6 structures (Scale bar $=200 \mathrm{~nm}$ ). (b) Rendered images of origami ROIs containing 1, 2, 3, 4, 5 and 6 detected TRAIL peptides (Scale bar $=50 \mathrm{~nm})$. 

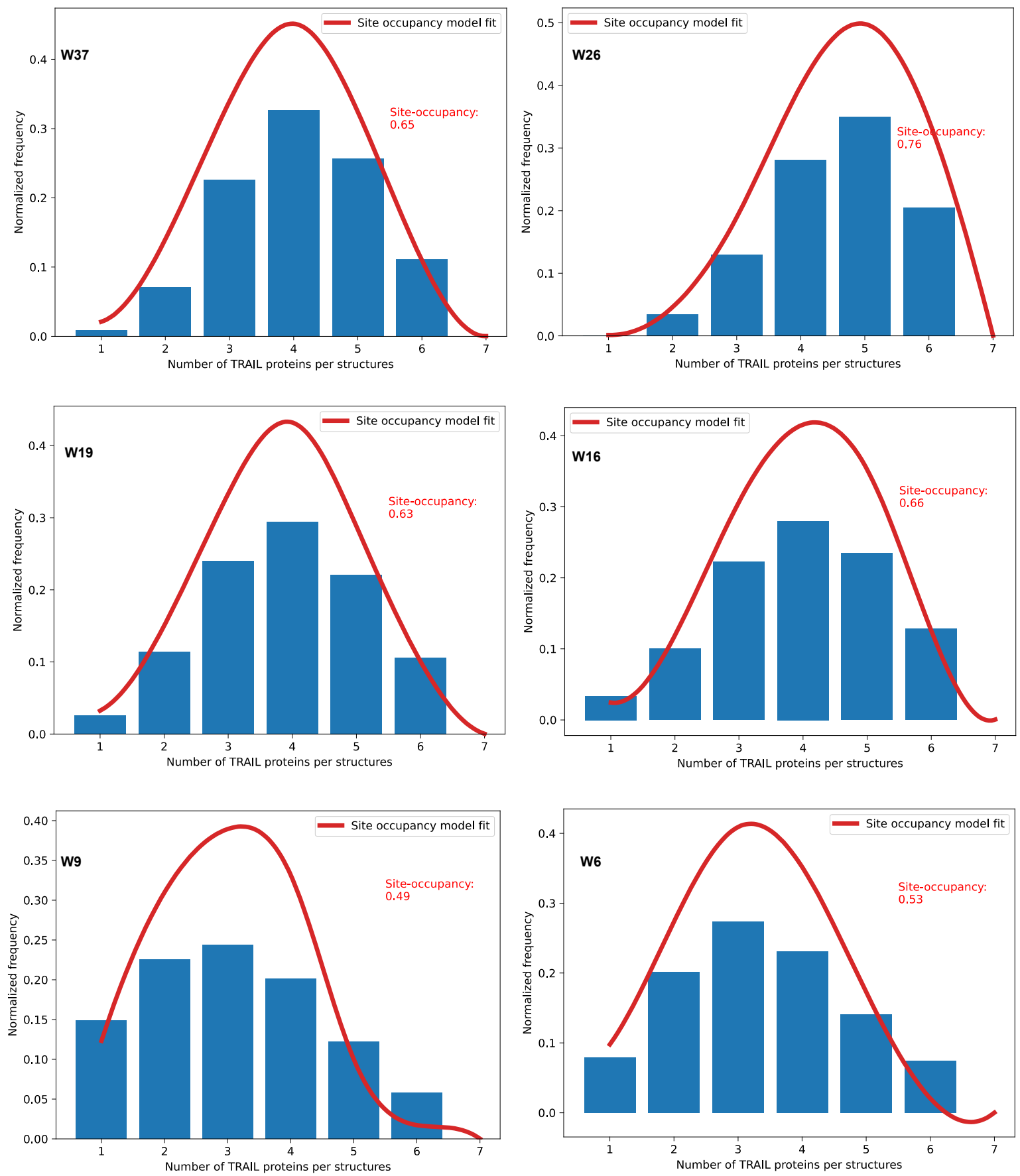

Figure S24. Protruding DNA site occupancy and the distribution of number of peptide per structure for each W-structure. This is computed from DNA-PAINT data. 

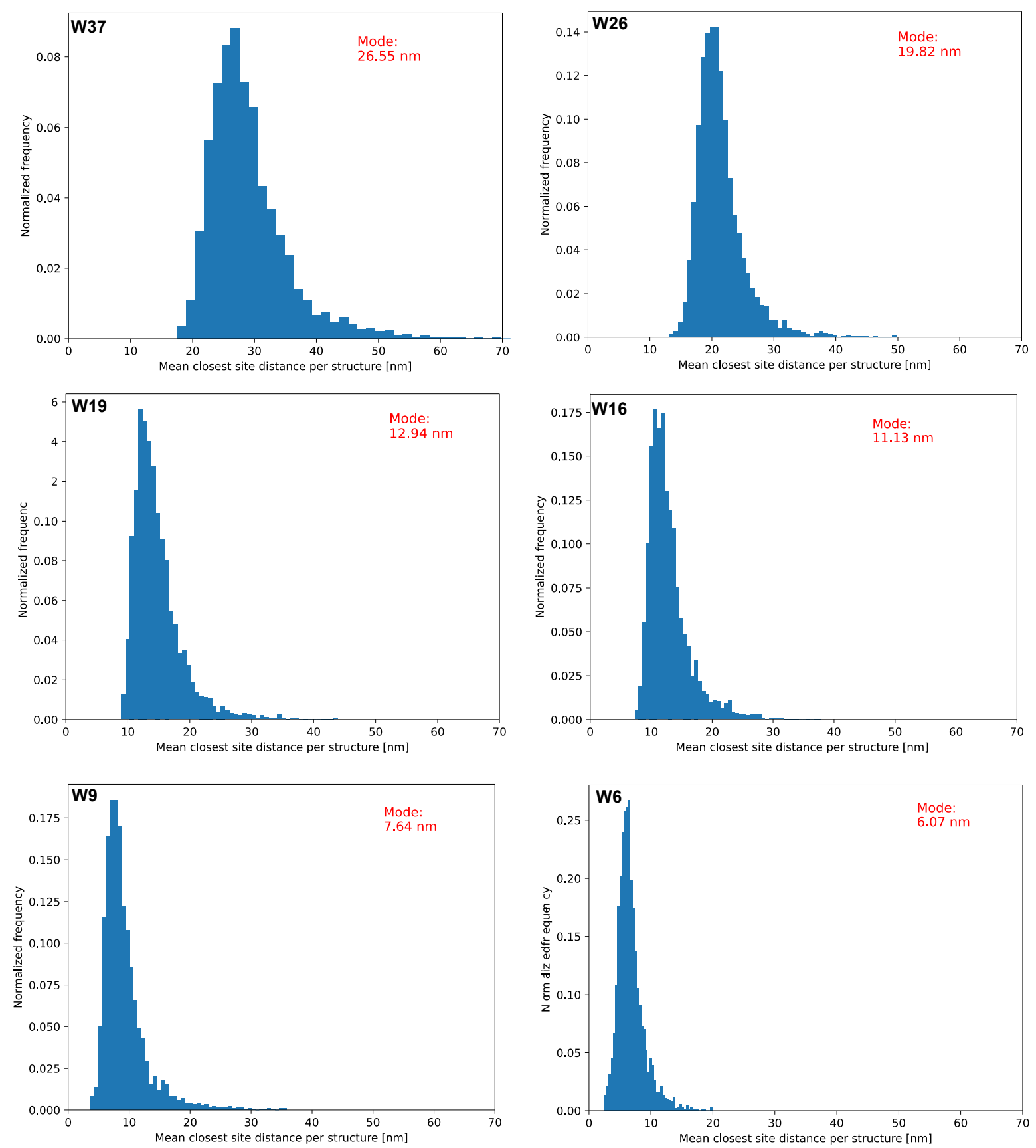

Figure S25. The inter-peptide distance distribution of each W-structure. This is computed from DNA-PAINT data. 
a

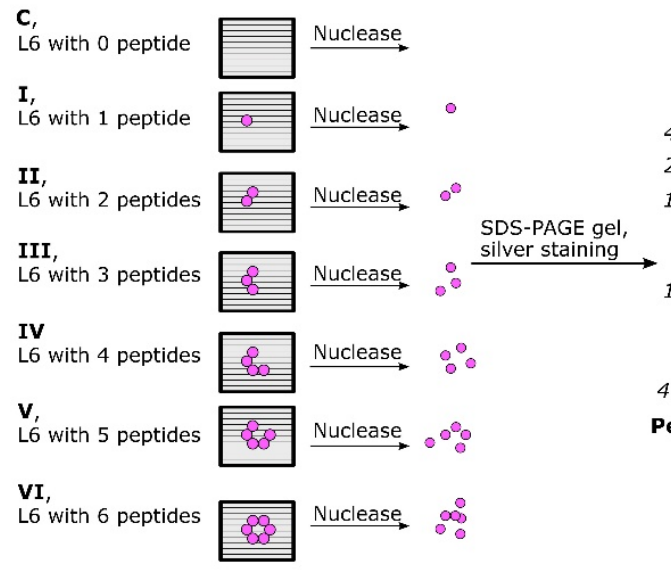

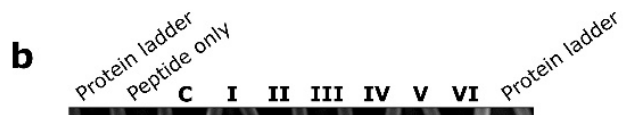

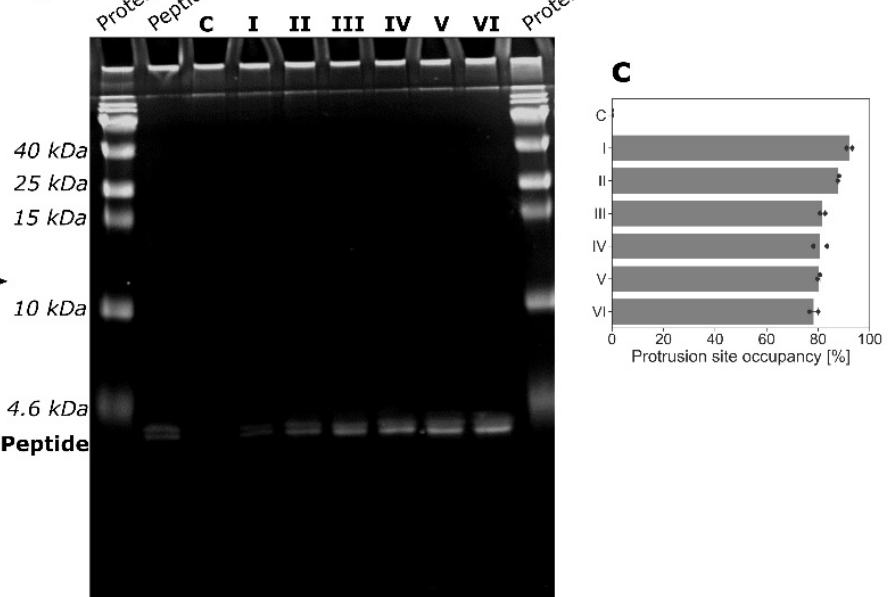

Figure S26. Gel-based peptide quantification of L6. L6 structures attached with different numbers of peptides (a) were prepared, then they were treated with DNase I to completely digest the DNA origami template. The samples were then run on SDS-PAGE (4-20\% gradient gel) gel. Silver staining was carried out to visualize the peptide (b). We did not see obvious DNase I band, which could be caused by its very low loading amount. The protrusion site occupancy calculated from the band intensities plotted in (c). 

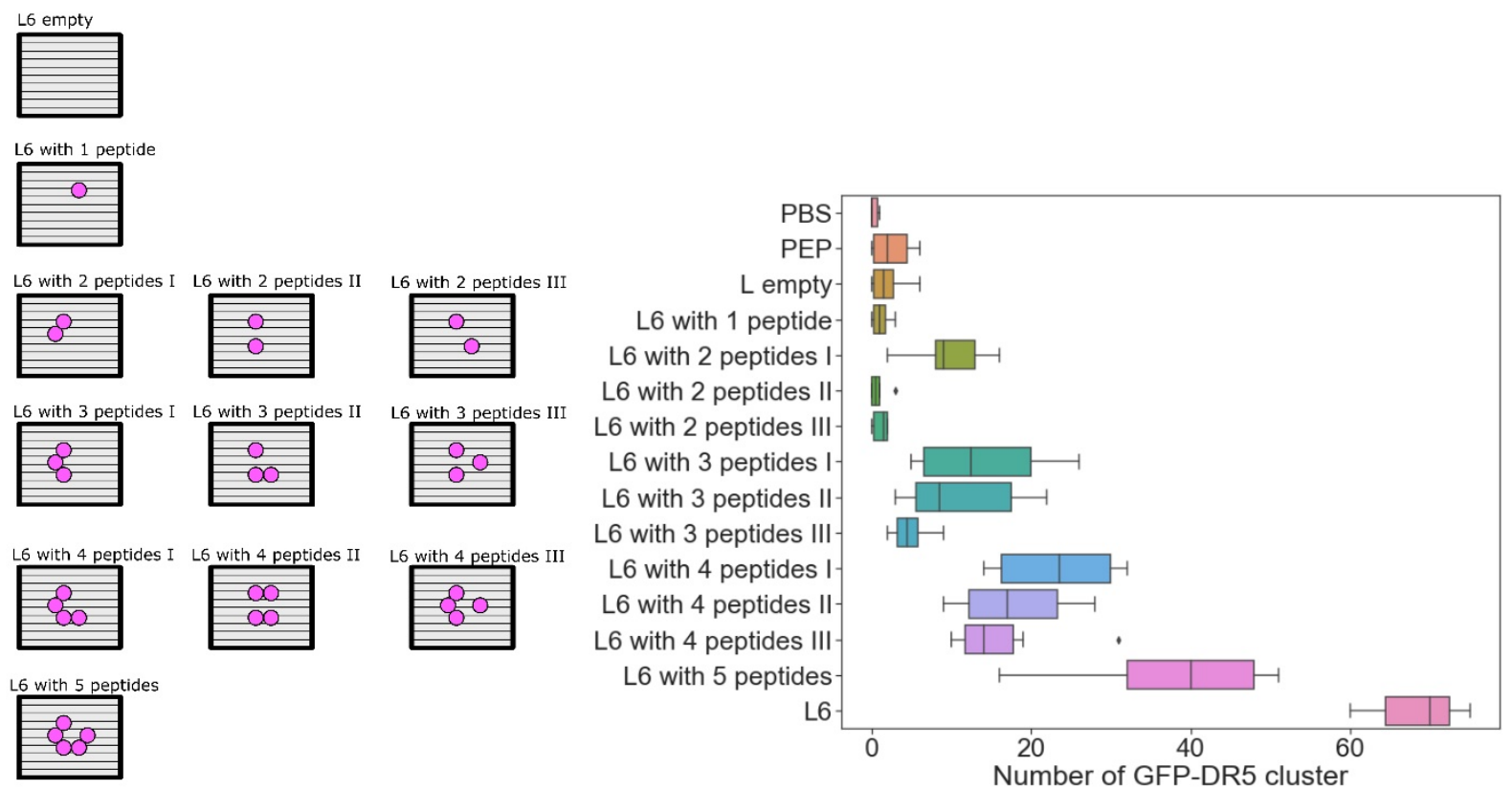

16

L6 with 6 peptides

L6 with 2 peptides

L6 with 2 peptides II

L6 with 2 peptides III

L6 with 3 peptides I

L6 with 3 peptides I

L6 with 3 peptides II

L6 with 4 peptides

L6 with 4 peptides II

6 with 4 peptides III

L6 with 5 peptides

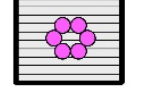

Figure S27. Quantification of GFP-DR5 clusters induced by L6 attached with different number

of peptides on GFP-DR5-expressing MCF-7 cell. Left: the illustration of peptide numbers and patterns; Right: GFP-DR5 cluster counting. $(\mathrm{n}=50$ cells $)$. The treatment was 4 hours with 2nM DNA origami structures (12-nM peptides). 


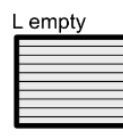

L6 with 1 peptide

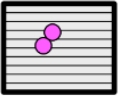

L6 with 2 peptides I L6 with 2 peptides II L6 with 2 peptides

L6 with 3 peptides I L6 with 3 peptides II L6 with 3 peptides III

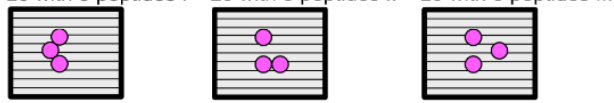

L6 with 4 peptides I L6 with 4 peptides II L6 with 4 peptides III

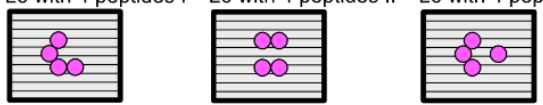

L6 with 5 peptides

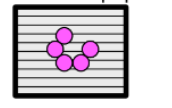

L6 with 6 peptides
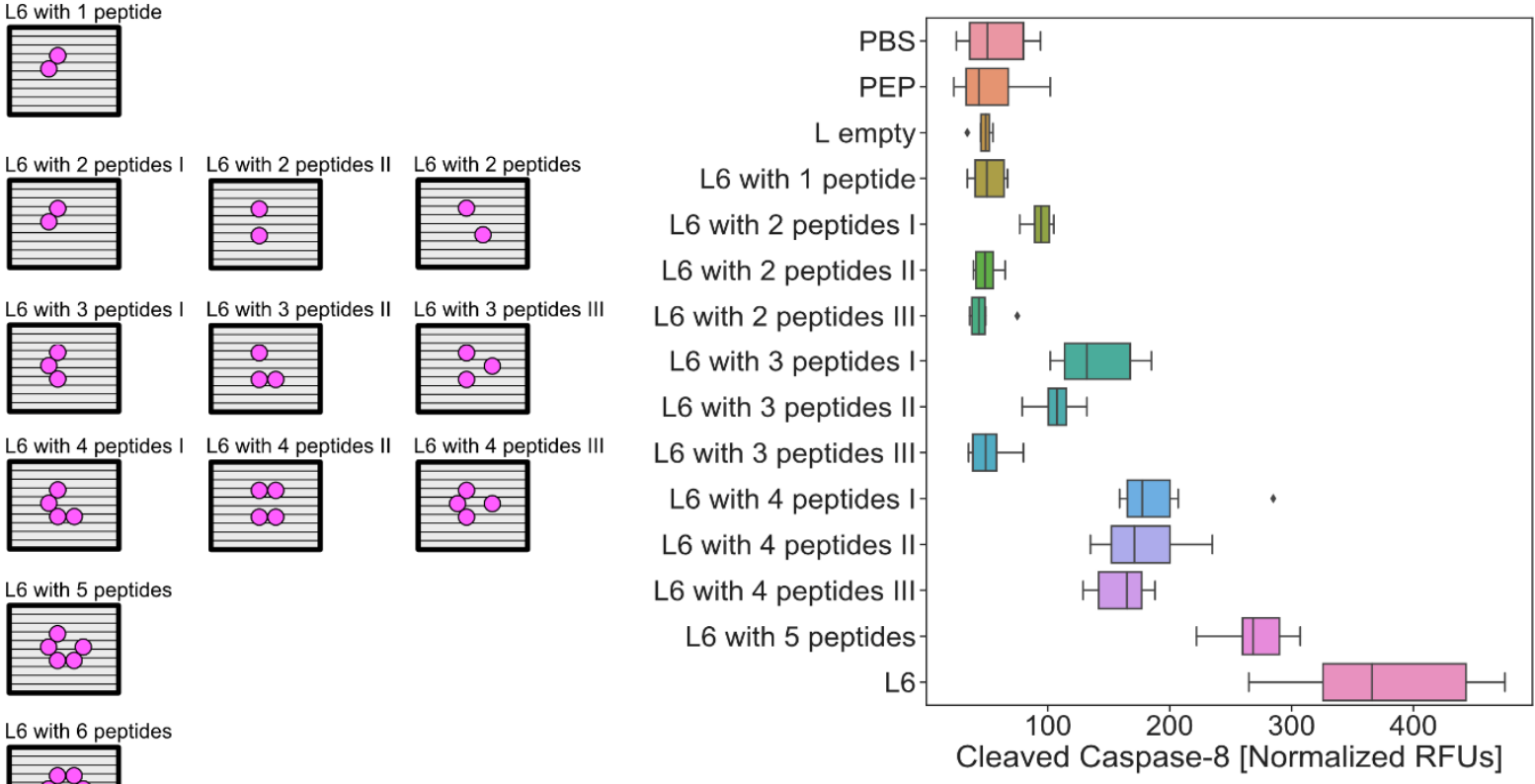

Figure S28. Normalized (to GAPDH) cleaved caspase-8 level induced by L6 attached with different number of peptides on GFP-DR5-expressing MCF-7 cell. Left: the illustration of peptide numbers and patterns; Right: Normalized (to GAPDH) cleaved caspase- 8 level. The treatment was 1.5 hours with 2-nM DNA origami structures (12-nM peptides). 
Extended Table.1| The half maximal inhibitory concentration $\left(\mathrm{IC}_{50}\right)$ of peptides.

\begin{tabular}{|l|l|l|l|}
\hline Treatment & IC $50[\mathrm{nM}]$ MCF-7 & IC $50[\mathrm{nM}]$ SK-BR-3 & IC50 [nM] MDA-MB-231 \\
\hline Peptide & $-^{[\mathrm{a}]}$ & $-^{[\mathrm{a}]}$ & $-^{[\mathrm{a}]}$ \\
\hline W & $-^{[\mathrm{b}]}$ & $-^{[\mathrm{b}]}$ & $-^{[\mathrm{b}]}$ \\
\hline W6 & 14.3 & 9.0 & 9.4 \\
\hline W9 & 52.7 & 44.2 & 68.3 \\
\hline W16 & $-^{[\mathrm{a}]}$ & $-^{[\mathrm{a}]}$ & $-^{[\mathrm{a}]}$ \\
\hline W19 & $-^{[\mathrm{a}]}$ & $-^{[\mathrm{a}]}$ & $-^{[\mathrm{a}]}$ \\
\hline W26 & $-^{[\mathrm{a}]}$ & $-^{[\mathrm{a}]}$ & $-^{[\mathrm{a}]}$ \\
\hline L & $-^{[\mathrm{b}]}$ & $-^{[\mathrm{b}]}$ & $-^{[\mathrm{b}]}$ \\
\hline L6 & 2.2 & 4.5 & 3.7 \\
\hline L11 & $-^{[\mathrm{b}]}$ & $-^{[\mathrm{b}]}$ & 292.7 \\
\hline
\end{tabular}

[a] Determined up to $300 \mathrm{nM}$ of peptide.

[b] Determined up to $50 \mathrm{nM}$ of DNA origami structures. 